\title{
Polyconvex energies and cavitation
}

\author{
Pietro Celada and Stefania Perrotta
}

\begin{abstract}
We study the existence of singular minimizers in the class of radial deformations for polyconvex energies that grow linearly with respect to the Jacobian.
\end{abstract}

Mathematics Subject Classification (2010). Primary 49J45; Secondary $74 \mathrm{~B} 20$.

Keywords. Polyconvex integrals, Radial singular minimizers, Cavitation.

\section{Introduction}

The aim of this paper is to study the existence of singular minimizers for variational integrals with polyconvex energies in the radially symmetric case.

The variational elasticity problem that motivates this investigation can be described as follows. Let the open unit ball $B_{1}$ in $\mathbb{R}^{N}$ be the reference configuration of a hyperelastic, isotropic material with stored energy density $W$ so that the total energy corresponding to a smooth deformation $u$ with given displacement $u(x)=\lambda x(\lambda>1)$ at the boundary $|x|=1$ is given by

$$
E(u)=\int_{B_{1}} W(D u(x)) d x .
$$

We assume that $W(D u) \rightarrow+\infty$ as $\operatorname{det} D u \rightarrow+\infty$ and $\operatorname{det} D u \rightarrow 0^{+}$. We restrict our analysis to the special case of radial deformations, i.e. deformations $u$ of the form $u(x)=v(|x|) x /|x|$ with $v(r)$ positive and strictly increasing and such that $v(1)=\lambda$, so that, by a change of variables, the total energy corresponding to $u$ becomes

$$
E(u)=J(v)=\sigma_{N} \int_{0}^{1} r^{N-1} \Phi\left(v^{\prime}(r), \frac{v(r)}{r}\right) d r
$$

Dedicated to A. Cellina on his seventieth birthday. 
where $\sigma_{N}$ is the $(N-1)$-dimensional measure of $\partial B_{1}$ and $\Phi$ is associated with the stored energy density $W$, see Sect. 2. We look for those radial deformations that minimize the total energy among all radial ones including those satisfying $v(0)>0$, i.e. corresponding to deformations $u$ which are singular at the origin. The existence of optimal radial deformations with $v(0)>0$ for large enough displacement $\lambda$ at the boundary can be interpreted as the occurrence of a spherical fracture - a cavity -inside the body, a behaviour which is actually observed in experiments with elastomers. This is the problem studied by Ball in his seminal paper [1], see also [8-10], just to mention a few other references. We mention also [4] for a description of cavitation in the language of currents and we refer to [5] for a survey of theoretical and experimental results about cavitation.

Among the results of [1], those which are relevant for our analysis regard isotropic, compressible materials whose stored energy density $W(D u)$ takes the form

$$
W(D u)=\theta(|D u|)+w(\operatorname{det} D u)
$$

where $\theta$ is a convex function with polynomial growth of order $1<p<N$ at infinity and $w$ is a strictly convex, superlinear function. Note that the growth assumption on $\theta$ allows for discontinuous deformations with finite energy. It was proved by Ball in [1] that there is a threshold $\lambda_{c}>1$ such that the linear function $v_{\lambda}(r)=\lambda r$ is the unique minimizer of $J$ for $1 \leq \lambda \leq \lambda_{c}$ whereas $J$ has a unique minimizer $v$ with $v(0)>0$ for $\lambda>\lambda_{c}$. The model considered by Ball thus predicts the occurrence of cavitation and seems to be in good agreement with some of the experimental results. Yet, the assumption of superlinearity with respect to det $D u$ is not consistent with some of the experimental results of [2] which suggest that cavitation occurs for isotropic, compressible materials whose energy density $W(D u)$ has linear growth with respect to $\operatorname{det} D u$ as $\operatorname{det} D u \rightarrow+\infty$.

Another important contribution to the study of cavitation was given by Marcellini in [6]. Marcellini's approach to the problem is based on the idea that, contrary to Ball's approach, the energy corresponding to a singular, radial deformation $v$ must be defined by lower semicontinuity or relaxation, i.e. choosing the energy of the radial deformation associated to $v$ to be

$$
J_{V}(v)=\inf \left\{\liminf _{k \rightarrow+\infty} J\left(v_{k}\right): v_{k} \rightarrow v\right\}
$$

where the greatest lower bound is taken among all regular deformations $v_{k}$, i.e. $v_{k}(0)=0$, and the convergence $v_{k} \rightarrow v$ is the natural weak convergence for which $J$ is lower semicontinuous, see Sect. 2. Marcellini's main result is the derivation of the following representation formula for the relaxed energy

$$
J_{V}(v)=\sigma_{N} \int_{0}^{1} r^{N-1} \Phi\left(v^{\prime}(r), \frac{v(r)}{r}\right) d r+w^{\infty} \frac{\sigma_{N}}{N}[v(0)]^{N} .
$$

Here, $\Phi$ comes from an energy density $W$ of the form (1.1) and the coefficient $w^{\infty}$ is the recession of the convex function $w$ at $t=1$. The additional term 
appearing in the relaxed energy $J_{V}$ is proportional to the $N$-dimensional measure of the cavity and can be interpreted as the contribution of the singular part of the Jacobian determinant of the radial deformation $u(x)=v(|x|) x /|x|$ to the total energy. It clearly penalizes the occurrence of cavitation and moreover, according to this model and contrary to Ball's, singular radial deformations may have finite energy only if the energy density $W$ in (1.1) grows linearly with respect to $\operatorname{det} D u \rightarrow+\infty$. This behaviour agrees with the experimental results of [2].

A further model for cavitation is studied by Müller and Spector in [7]. They address the full 3D problem for an energy density which includes a surface term which accounts for the energy required for the creation of new surfaces and which has superlinear growth with respect to det $D u \rightarrow+\infty$. The deep analysis of [7] shows that minimizers for this model exist and that cavitation is allowed. Yet, it seems to us that it is not proved that it actually occurs, even in the simplified case of radial deformations.

We now come to the content of this paper. We investigate the existence of radial, singular minimizers for an energy $J_{S}$ which includes a surface term. The energy $J_{S}$ we consider here is a special instance of the full $3 \mathrm{D}$ energy considered in [7], the differences being that we consider radial deformations only and that the energy density is supposed to grow linearly with respect to $\operatorname{det} D u \rightarrow+\infty$. It is given by

$$
J_{S}(v)=\sigma_{N} \int_{0}^{1} r^{N-1} \Phi\left(v^{\prime}(r), \frac{v(r)}{r}\right) d r+w^{\infty} \sigma_{N}[v(0)]^{N-1}
$$

where $\Phi$ and $w^{\infty}$ are the same as in $J_{V}$.

Preliminary to this investigation, we give an explicit proof that the linear function $v_{\lambda}$ is the unique minimizer of $J_{V}$ for every $\lambda \geq 1$. Thus, the relaxed energy $J_{V}$ does not allow for cavitation. Indeed, the minimality of the linear function $v_{\lambda}$ for $J_{V}$ is a somewhat expected result, compare the discussion in [6]. As to this issue, we mention also Theorem 3 in 2.6.3 of [4], though it seems to us that the proof given is not correct.

Then, we consider the energy $J_{S}$ and we prove that, though it is not lower semicontinuous for the natural weak convergence associated with the problem, yet minimizers of $J_{S}$ exist for every $\lambda>1$. This is established by computing the relaxation of $J_{S}$ in the spirit of Marcellini's approach (Theorem 4.1) and showing that $J_{S}$ and its relaxation agree at every minimizer of the latter. We then prove that minimizers of $J_{S}$ are singular for large enough $\lambda>1$, i.e. cavitation occurs. In fact, since $v_{\lambda}$ turns out to be the unique solution to the Euler-Lagrange equation for $J_{S}$ with $v(0)=0$ and for large enough $\lambda>1$ there are functions $v$ with $v(0)>0$ such that $J_{S}(v)<J_{S}\left(v_{\lambda}\right)$, we conclude that $J_{S}$ has singular minimizers for large enough $\lambda>1$.

Finally, we present some explicit computations for the radial, 3D model case whose energy density is

$$
W(A)=|A|^{2}+\operatorname{det} A+\frac{1}{\operatorname{det} A}, \quad A \in \mathbb{M}^{3 \times 3} \text { with } \operatorname{det} A>0 .
$$


In this case, we prove that there are critical values $1<\lambda_{c}^{-} \leq \lambda_{c}^{+}$such that the linear function $v_{\lambda}$ is the unique minimizer of $J_{S}$ for $\lambda<\lambda_{c}^{-}$whereas $J_{S}$ has a singular minimizer for every $\lambda>\lambda_{c}^{+}$. For $\lambda$ in the possible, intermediate range between $\lambda_{c}^{-}$and $\lambda_{c}^{+}$, our analysis only proves that, besides the linear function $v_{\lambda}$, there are other solutions to the Euler-Lagrange equation of $J_{S}$ which are singular at $r=0$ but does not yield any information whether the minimizer is the linear function $v_{\lambda}$ or any of the singular solutions, though the obvious conjecture is that $\lambda_{c}^{-}=\lambda_{c}^{+}$.

\section{Notation and description of the problem}

Notations. We denote the norm of a vector $x$ in $\mathbb{R}^{N}$ by $|x|$. If $A$ is a subset of $\mathbb{R}^{N}$, we denote the interior, the closure and the boundary of $A$ by $\operatorname{int}(A), \bar{A}$ and $\partial A$ respectively.

As to matrices, let $\mathbb{M}^{N \times N}$ be the set of all $N \times N$ real matrices $A=$ $\left(A_{n}^{m}\right)_{m, n=1, \ldots, N}$ endowed with the Euclidean norm denoted by $|A|$ and let $\mathbb{I}_{N}$ be the identity matrix. The singular values of the matrix $A$ are the eigenvalues $\lambda_{1}(A), \ldots, \lambda_{N}(A)$ of the positive, symmetric matrix $\sqrt{A A^{T}}$ so that

$$
|A|^{2}=\lambda_{1}^{2}(A)+\cdots+\lambda_{N}^{2}(A) \quad \text { and } \quad|\operatorname{det} A|=\lambda_{1}(A) \cdots \lambda_{N}(A) .
$$

The standard basis of $\mathbb{R}^{N}$ is denoted by $\left\{e_{1}, \ldots, e_{N}\right\}$ and the tensor product of two vectors $a=a^{1} e_{1}+\cdots+a^{N} e_{N}$ and $b=b^{1} e_{1}+\cdots+b^{N} e_{N}$ is the rankone matrix $a \otimes b$ defined by $(a \otimes b)_{n}^{m}=a^{m} b^{n}$ for every $m$ and $n$. Finally, we denote the group of all matrices with positive determinant by $\mathbb{M}_{+}^{N \times N}$ and the subgroup of special orthogonal matrices by $S O(N)$.

As regards measure and functional theoretic notation, we denote the Lebesgue measure of a measurable subset $E$ in the Euclidean space $\mathbb{R}^{n}$ by $|E|$. We use standard notation for the spaces of continuously differentiable functions and for Lebesgue and Sobolev spaces and their norms. In the special case of functions of one variable on a bounded interval $I$, we let $A C(I)$ and $A C_{\text {loc }}(I)$ be the spaces of absolutely continuous functions on $I$ and on all compact subintervals of $I$ respectively.

The variational problem. As explained in the introduction, we are interested in the deformations of a hyperelastic, homogeneous, solid body whose reference configuration is the open unit ball $B_{1}$ of $\mathbb{R}^{N}$, the physically interesting cases being obviously $N=2$ and $N=3$. We assume that the stored energy density of the body is a nonnegative, smooth, strictly polyconvex function $W$ which is the sum of two terms:

$$
W(A)=\theta(|A|)+w(\operatorname{det} A), \quad A \in \mathbb{M}_{+}^{N \times N} .
$$

For the radially symmetric term $\theta$, we assume the following hypotheses:

(H1) $\theta \in \mathcal{C}^{3}([0,+\infty))$ and $\theta \geq 0$;

(smoothness and positivity)

(H2) $\theta$ is strictly convex with $\theta^{\prime}(0)=0$;

(convexity)

so that $A \in \mathbb{M}^{N \times N} \mapsto \theta(|A|)$ is strictly convex as well and

(H3) $t^{p} \leq \theta(t) \leq C\left(1+t^{p}\right)$ for every $t \geq 0$;

(growth and coercivity) 
for some constant $C>0$ and some index $1<p<N$ so that possibly discontinuous deformations might have finite energy. Note also that (H2) and (H3) imply that

$$
0 \leq \theta^{\prime}(t) \leq C_{1}\left(1+t^{p-1}\right) \leq C_{2}\left[1+\frac{\theta(t)}{t}\right], \quad t \geq 0 .
$$

As to the term $w$ depending on the deformation of volume elements, we assume the following hypotheses:

(H4) $w \in \mathcal{C}^{3}((0,+\infty))$ and $w \geq 0$;

(smoothness and positivity)

(H5) $w$ is convex on $(0,+\infty)$;

(convexity)

(H6) $w(t) \rightarrow+\infty$ as $t \rightarrow 0^{+}$;

(behavior at zero)

(H7) there is $\delta>0$ and $C \geq 0$ such that

$$
\left|(s t) w^{\prime}(s t)\right| \leq C w(t), \quad t>0,
$$

for every $|s-1| \leq \delta$;

(H8) $w(t) / t \rightarrow w^{\infty} \in(0,+\infty)$ as $t \rightarrow+\infty$.

(linear growth)

Note that the hypotheses (H5) and (H6) imply that $w^{\prime}(t) \rightarrow-\infty$ as $t \rightarrow 0^{+}$ and that (H8) expresses the property that $w$ has linear growth at infinity. In the superlinear case, one would have $w^{\infty}=+\infty$. As regards the hypothesis (H7), it is a structure hypothesis on $w$ which is satisfied for instance by suitable perturbations of the model case $w(t)=t+1 / t^{\alpha}, t>0(\alpha>0)$. As mentioned in the introduction, the model case of the energy density $W$ we have in mind is given by (1.2) in dimension $N=3$.

For this energy density $W$, the total energy associated with a smooth deformation $u: B_{1} \rightarrow \mathbb{R}^{N}$ is given by the integral

$$
E(u)=\int_{B_{1}} W(D u(x)) d x .
$$

Yet, we want to consider here bounded deformations which are possibly singular at the origin, i.e. deformations $u$ corresponding to possibly discontinuous Sobolev functions $u \in L^{\infty}\left(B_{1}, \mathbb{R}^{N}\right) \cap W^{1,1}\left(B_{1}, \mathbb{R}^{N}\right)$ satisfying an appropriate notion of invertibility. The definition of the appropriate notion of invertibility for irregular Sobolev mappings is delicate and we refer to the book by Giaquinta, Modica and Souček [3] and the paper by Müller and Spector [7] for a general discussion of this issue. Here, we take advantage of the fact that we shall consider the variational problem for $E$ only in the restricted class of radial deformations where a sensible definition of invertibility can be stated in the most elementary terms.

In fact, we shall consider the class of radial deformations for which no eversion occurs, i.e. the class of all mappings $u \in L^{\infty}\left(B_{1}, \mathbb{R}^{N}\right)$ such that

$$
u(x)=v(|x|) \frac{x}{|x|} \quad \text { for a.e. } x \in B_{1}
$$

for some $v \in L^{\infty}(0,1)$ satisfying $v>0$ almost everywhere on $(0,1)$. It is clear that $v$ is uniquely associated with $u$ up to a null set by (2.4) and viceversa. It is then easy to check (see Lemma 4.1 in [1]) that, whenever the two measurable 
functions $u: B_{1} \rightarrow \mathbb{R}^{N}$ and $v:(0,1] \rightarrow[0,+\infty)$ are related by (2.4), we have that

$$
u \in W^{1, p}\left(B_{1}, \mathbb{R}^{N}\right) \Longleftrightarrow\left\{\begin{array}{l}
v \in A C_{\mathrm{loc}}((0,1]) \\
\int_{0}^{1} r^{N-1}\left(\left|v^{\prime}(r)\right|^{p}+\left|\frac{v(r)}{r}\right|^{p}\right) d r<+\infty
\end{array}\right.
$$

for every index $1 \leq p<+\infty$. In this case, the gradient of $u$ and its singular values are given by

$$
D u(x)=\frac{v(|x|)}{|x|} \mathbb{I}_{N}+\left(v^{\prime}(|x|)-\frac{v(|x|)}{|x|}\right) \frac{x \otimes x}{|x|^{2}} \quad \text { for a.e. } x \in B_{1}
$$

and

$$
\left\{\begin{array}{l}
\lambda_{1}(D u(x))=v^{\prime}(|x|) \\
\lambda_{n}(D u(x))=\frac{v(|x|)}{|x|} \quad n=2, \ldots, N \quad \text { for a.e. } x \in B_{1} .
\end{array}\right.
$$

It follows from (2.5) that a singular, radial deformation $u$ with $v(0)>0$ cannot be in $W^{1, p}\left(B_{1}, \mathbb{R}^{N}\right)$ for $p \geq N$.

We shall assume throughout the paper that $u$ defined by (2.4) is such that the corresponding $v$ can be chosen to be strictly increasing. Thus, $u$ is injective and $v$ is actually defined up to a countable set and we assume also that it is defined by continuity at $r=0$ and $r=1$. With this additional assumption, it follows easily that the equivalence (2.5) actually holds with $v \in A C([0,1])$ and moreover, for these mappings $u$ satisfying (2.4) and (2.5) for some $p \geq 1$, the distributional Jacobian determinant is a nonnegative Radon measure whose absolutely continuous part with respect to the Lebesgue measure has density

$$
\operatorname{det} D u(x)=v^{\prime}(|x|)\left(\frac{v(|x|)}{|x|}\right)^{N-1} \quad \text { for a.e. } x \in B_{1}
$$

and whose singular part is

$$
(\operatorname{Det} D u)^{\mathrm{s}}=\frac{\sigma_{N}}{N}[v(0)]^{N} \delta_{0}
$$

where $\delta_{0}$ is the Dirac measure at the origin.

For the energy $E$ defined by (2.3), we shall consider the radial displacement boundary value problem in the class of radial deformations, i.e. the variational problem of minimizing the energy $E$ among all radial deformations $u$ in $L^{\infty}\left(B_{1}, \mathbb{R}^{N}\right) \cap W^{1,1}\left(B_{1}, \mathbb{R}^{N}\right)$ satisfying $\operatorname{det} D u>0$ almost everywhere on $B_{1}$ and the boundary condition $u(x)=\lambda x$ for $|x|=1$ for some $\lambda>1$. The set of all functions $v$ associated with these radial deformations $u$ by (2.4) is the set

$$
\mathcal{A}=\left\{v \in A C([0,1]): v>0 \text { on }(0,1] \text { and } v^{\prime}>0 \text { a.e. on }(0,1]\right\}
$$

and we denote by $\mathcal{A}(\lambda)$ those $v \in \mathcal{A}$ such that $v(1)=\lambda$. Note also that the second condition in (2.5) yields

$$
\int_{0}^{1} r^{N-1}\left[v^{\prime}(r)+\frac{v(r)}{r}\right] d r<+\infty
$$


for every $v \in \mathcal{A}(\lambda)$. By a change of variables and by (2.1) and (2.7), we obtain

$$
E(u)=\sigma_{N} \int_{0}^{1} r^{N-1} \Phi\left(v^{\prime}(r), \frac{v(r)}{r}\right) d r
$$

where

$$
\Phi(\xi, \eta)=\Phi^{1}(\xi, \eta)+\Phi^{2}(\xi, \eta)=\theta\left(\sqrt{\xi^{2}+(N-1) \eta^{2}}\right)+w\left(\xi \eta^{N-1}\right)
$$

for every $\eta, \xi>0$. We note that the first term $\Phi^{1}$ corresponding to the radially symmetric part of $W$ is actually defined on $\mathbb{R} \times \mathbb{R}$ and its properties can be easily read from the corresponding properties of $\theta$, i.e.

$\left(H 1^{\prime}\right) \Phi^{1} \in \mathcal{C}^{3}(\mathbb{R} \times \mathbb{R})$ and $\Phi^{1} \geq 0 ;$

$\left(\mathrm{H} 2^{\prime}\right) \Phi^{1}$ is strictly convex on $\mathbb{R} \times \mathbb{R}$ and $D \Phi^{1}(0,0)=0$;

$\left(\mathrm{H}^{\prime}\right) \xi^{p} \leq \Phi^{1}(\xi, \eta) \leq C\left(1+\xi^{p}+\eta^{p}\right)$ for every $(\xi, \eta) \in \mathbb{R} \times \mathbb{R}$.

We denote the partial derivatives of $\Phi$ by $\Phi_{\eta}, \Phi_{\xi}, \Phi_{\xi \xi}$ and so on and similarly for $\Phi^{i}$. For future purposes, we record the following estimates for the derivatives of $\Phi^{i}$. As regards $\Phi^{1}$, we easily obtain from (2.2) that

$$
\left|D \Phi^{1}(\xi, \eta)\right| \leq C\left[1+\frac{1}{|\eta|} \Phi^{1}(\xi, \eta)\right] \quad \text { for } \eta \neq 0, \xi \in \mathbb{R}
$$

holds for some constant $C=C(N)$ or either that, for every $L>0$, there is a constant $C=C(N, L)$ such that

$$
\left|D \Phi^{1}(\xi, \eta)\right| \leq C\left(1+|\xi|^{p-1}\right) \quad|\eta| \leq L, \xi \in \mathbb{R} .
$$

As to the derivatives of $\Phi^{2},(\mathrm{H} 7)$ yields $\delta>0$ such that

$$
\begin{aligned}
& \left|\Phi_{\eta}^{2}(\xi, \bar{\eta})\right| \leq \frac{C}{\eta} \Phi^{2}(\xi, \eta) \quad \text { for } \eta, \bar{\eta}>0 \text { and }|\bar{\eta} / \eta-1| \leq \delta \\
& \left|\Phi_{\xi}^{2}(\xi, \eta)\right| \leq \frac{C}{\xi} \Phi^{2}(\xi, \eta) \quad \text { for } \xi, \eta>0
\end{aligned}
$$

for some constant $C=C(N, \delta)$.

Going back to the variational problem, we are thus led to consider the integral

$$
J(v)=\sigma_{N} \int_{0}^{1} r^{N-1} \Phi\left(v^{\prime}(r), \frac{v(r)}{r}\right) d r, \quad v \in \mathcal{A}(\lambda),
$$

and the associated variational problem

$$
\min \{J(v): v \in \mathcal{A}(\lambda)\} .
$$

The convergence considered in the set of admissible, radial deformations $\mathcal{A}(\lambda)$ is the natural convergence induced on minimizing sequences of $J$ : if $v_{k} \in \mathcal{A}(\lambda)$ for every $k$ and $v \in \mathcal{A}(\lambda), v_{k} \rightarrow v$ weakly in $\mathcal{A}(\lambda)$ means that

$$
\begin{cases}v_{k} \rightarrow v & \text { pointwise on }(0,1] ; \\ v_{k}^{\prime} \rightarrow v^{\prime} & \text { weakly in } L^{1}(\varepsilon, 1) \text { for every } 0<\varepsilon<1\end{cases}
$$


Then, $J$ is sequentially lower semicontinuous with respect to this weak convergence by standard results. Moreover, it is easy to check that $\mathcal{A}(\lambda)$ is not closed for this weak convergence but the sublevel sets $\{J \leq c\}$ are sequentially compact for this weak convergence.

\section{Marcellini's relaxed model}

In this section, following Marcellini's approach to the problem of cavitation described in [6], we consider the relaxation $J_{V}$ of $J$ on $\mathcal{A}(\lambda)$ defined by (3.1) below and we prove that the linear function $v_{\lambda}(r)=\lambda r$ for $0<r \leq 1$ is the unique minimizer of $J_{V}$ for every $\lambda \geq 1$. Therefore, the relaxed integral $J_{V}$ does not account for cavitation. This conclusion follows from the following claims.

Integral representation of $\boldsymbol{J}_{\boldsymbol{V}}$. The integral representation of $J_{V}$ defined by

$$
J_{V}(v)=\inf \left\{\liminf _{k} J\left(v_{k}\right): v_{k} \in \mathcal{A}(\lambda), v_{k}(0)=0 \text { and } v_{k} \rightarrow v\right\}
$$

where $J$ is defined by (2.17) and $\Phi$ is associated to the energy density $W$ satisfying (H1), (H2), (H3) and (H5) is a special case of Marcellini's result, see Theorem 1 in [6]. Marcellini's result reads as follows.

Theorem 3.1. Assume that (H1), (H2), (H3) and (H5) hold. Then,

$$
J_{V}(v)=J(v)+w^{\infty} \frac{\sigma_{N}}{N}[v(0)]^{N}, \quad v \in \mathcal{A}(\lambda) .
$$

We recall that $w^{\infty}$ is the recession of $w$ at $t=1$, i.e. the limit of $w(t) / t$ as $t \rightarrow+\infty$ appearing in (H8). The additional term appearing in $J_{V}$ is thus proportional to the volume of the cavity and, in this model and contrary to Ball's, singular radial deformations require infinite energy for superlinear $w$.

The linear function $\boldsymbol{v}_{\boldsymbol{\lambda}}$ is a minimizer of $\boldsymbol{J}_{\boldsymbol{V}}$. The sublevel sets $\{J \leq c\}$ are closed and sequentially compact for the weak convergence defined by $(2.18)$ and the relaxed functional $J_{V}$ is sequentially lower semicontinuous along weakly converging sequences of functions in $\mathcal{A}(\lambda)$ by construction. Thus, existence of minimizers of $J_{V}$ on $\mathcal{A}(\lambda)$ follows from direct methods and we claim that the linear function $v_{\lambda}$ is a minimizer.

Theorem 3.2. Assume that (H1), (H2), (H3) and (H5) hold. Then,

$$
J_{V}(v) \geq J_{V}\left(v_{\lambda}\right), \quad v \in \mathcal{A}(\lambda) .
$$

Proof. It is enough to prove the "quasiconvexity" of the functional $J$ on nonsingular function $v$, i.e.

$$
J(v) \geq J\left(v_{\lambda}\right), \quad v \in \mathcal{A}(\lambda), v(0)=0,
$$

because the very definition (3.1) of the relaxed functional $J_{V}$ then immediately yields that $J_{V}(v) \geq J\left(v_{\lambda}\right)=J_{V}\left(v_{\lambda}\right)$ for every $v \in \mathcal{A}(\lambda)$. To prove (3.2), let $v \in \mathcal{A}(\lambda)$ be regular at $r=0$, i.e. $v(0)=0$, and let $u$ and $u_{\lambda}$ be the deformations 
of $B_{1}$ corresponding to $v$ and $v_{\lambda}$ respectively. Then, $u \in u_{\lambda}+W_{0}^{1,1}\left(B_{1}, \mathbb{R}^{N}\right)$ and for the first summand of $J$ we have

$$
\begin{gathered}
\sigma_{N} \int_{0}^{1} r^{N-1} \Phi^{1}\left(v^{\prime}, \frac{v}{r}\right) d r=\int_{B_{1}} \theta(|D u|) d x \\
\geq \frac{\sigma_{N}}{N} \theta\left(\left|\lambda \mathbb{I}_{N}\right|\right)=\sigma_{N} \int_{0}^{1} r^{N-1} \Phi^{1}\left(v_{\lambda}^{\prime}, \frac{v_{\lambda}}{r}\right) d r
\end{gathered}
$$

by Jensen's inequality. As to the second summand of $J$, choosing $0<\varepsilon<1$ and exploiting Jensen's inequality again, we obtain

$$
\begin{aligned}
& \sigma_{N} \int_{\varepsilon}^{1} r^{N-1} w\left(v^{\prime}\left(\frac{v}{r}\right)^{N-1}\right) d r \geq \sigma_{N} \frac{1-\varepsilon^{N}}{N} w\left(\frac{N}{1-\varepsilon^{N}} \int_{\varepsilon}^{1} v^{\prime} v^{N-1} d r\right) \\
& =\sigma_{N} \frac{1-\varepsilon^{N}}{N} w\left(\frac{\lambda^{N}-[v(\varepsilon)]^{N}}{1-\varepsilon^{N}}\right) .
\end{aligned}
$$

Letting $\varepsilon \rightarrow 0^{+}$and recalling that $v(0)=0$, we conclude that

$\sigma_{N} \int_{0}^{1} r^{N-1} w\left(v^{\prime}\left(\frac{v}{r}\right)^{N-1}\right) d r \geq \frac{\sigma_{N}}{N} w\left(\lambda^{N}\right)=\sigma_{N} \int_{0}^{1} r^{N-1} w\left(v_{\lambda}^{\prime}\left(\frac{v_{\lambda}}{r}\right)^{N-1}\right) d r$

and this together with (3.3) yields (3.2).

We remark that the "quasiconvexity" inequality (3.2) is not a straightforward consequence of the polyconvexity of the stored energy density $W$ of $E$ because the deformation $u$ corresponding to $v \in \mathcal{A}(\lambda)$ is in $W^{1,1}\left(B_{1}, \mathbb{R}^{N}\right)$ but need not be in $W^{1, N}\left(B_{1}, \mathbb{R}^{N}\right)$ even if $v(0)=0$.

The Euler-Lagrange equation for $\boldsymbol{J}_{\boldsymbol{V}}$. We now explore the optimality conditions satisfied by minimizers of $J_{V}$. The main issue in the derivation of the corresponding Euler-Lagrange (EL) equation lies in the fact that admissible variations have to comply with the constraint $v^{\prime}>0$ almost everywhere on $(0,1)$. This issue can be dealt with by exploiting essentially the same arguments of Theorem 7.3 in [1]. It can be useful to outline the main steps of this argument as the same reasoning will apply also to the EL equation of $J_{S}$.

Theorem 3.3. Assume that $(\mathrm{H} 1), \ldots,(\mathrm{H} 8)$ hold and let $v \in \mathcal{A}(\lambda)$ be a minimizer of $J_{V}$. Then,

(a) the mapping $r \in(0,1] \mapsto r^{N-2} \Phi_{\eta}\left(v^{\prime}, v / r\right)$ is in $L_{\text {loc }}^{1}((0,1])$;

(b) the mapping $r \in(0,1] \mapsto r^{N-1} \Phi_{\xi}\left(v^{\prime}, v / r\right)$ is in $A C_{\mathrm{loc}}((0,1])$;

(c) the equation

$$
\frac{\mathrm{d}}{\mathrm{d} r}\left[r^{N-1} \Phi_{\xi}\left(v^{\prime}, v / r\right)\right]=r^{N-2} \Phi_{\eta}\left(v^{\prime}, v / r\right)
$$

holds for a.e. $r \in(0,1]$;

(d) for every $\varepsilon \in(0,1)$ there exists $m=m(\varepsilon)>0$ such that $1 / m \leq v^{\prime}(r) \leq m$ for a.e. $r \in[\varepsilon, 1]$. 
Proof. From the estimates (2.13) and (2.15) with $\bar{\eta}=\eta$, we obtain

$$
\left|\Phi_{\eta}(\xi, \eta)\right| \leq C\left[1+\frac{1}{\eta} \Phi(\xi, \eta)\right], \quad \eta>0, \xi \in \mathbb{R} .
$$

Hence, picking $0<r_{0}<1$ and exploiting this with $\xi=v^{\prime}(r)$ and $\eta=v(r) / r$ for $r_{0} \leq r \leq 1$, we conclude that

$$
\int_{r_{0}}^{1}\left|\Phi_{\eta}\left(v^{\prime}, \frac{v}{r}\right)\right| r^{N-2} d r \leq C\left[1+\frac{1}{v\left(r_{0}\right)} \int_{r_{0}}^{1} \Phi\left(v^{\prime}, \frac{v}{r}\right) r^{N-1} d r\right]<+\infty
$$

and this proves (a).

To prove (b), consider the sets $E_{k}=\left\{r \in[1 / k, 1]: 1 / k \leq v^{\prime}(r) \leq k\right\}$ for $k \geq 1$, choose any function $\psi \in L^{\infty}(0,1)$ supported on $E_{k}$ and having zero average over the set $E_{k}$ itself, i.e.

$$
\psi=0 \text { a.e. on }[0,1] \backslash E_{k} \text { and } \int_{E_{k}} \psi d r=0
$$

and consider the variations

$$
v_{\varepsilon}(r)=v(r)+\varepsilon \int_{0}^{r} \psi d \rho, \quad 0 \leq r \leq 1 .
$$

Here, $\varepsilon \neq 0$ need not be positive. By the very definition of $E_{k}$, the functions $v_{\varepsilon}$ are admissible deformations for sufficiently small $|\varepsilon|$.

Now, we compute the (rescaled) differential quotient of $J_{V}$ at $v$ with increment $v_{\varepsilon}-v$ which, in view of the equality $v_{\varepsilon}(0)=v(0)$, coincides with the (rescaled) differential quotient of $J$. Then, by the mean value theorem, we have

$$
\begin{aligned}
& \frac{J_{V}\left(v_{\varepsilon}\right)-J_{V}(v)}{\varepsilon \sigma_{N}}=\int_{0}^{1} \frac{\Phi\left(v_{\varepsilon}^{\prime}, v_{\varepsilon} / r\right)-\Phi\left(v^{\prime}, v / r\right)}{\varepsilon} r^{N-1} d r \\
& =\int_{0}^{1}\left[\frac{\Phi\left(v_{\varepsilon}^{\prime}, v_{\varepsilon} / r\right)-\Phi\left(v^{\prime}, v_{\varepsilon} / r\right)}{\varepsilon}+\frac{\Phi\left(v^{\prime}, v_{\varepsilon} / r\right)-\Phi\left(v^{\prime}, v / r\right)}{\varepsilon}\right] r^{N-1} d r \\
& =\int_{0}^{1}\left[\Phi_{\xi}\left(\theta_{\varepsilon}^{1}, \frac{v_{\varepsilon}}{r}\right) \psi+\Phi_{\eta}\left(v^{\prime}, \frac{\theta_{\varepsilon}^{2}}{r}\right) \frac{1}{r} \int_{0}^{r} \psi d \rho\right] r^{N-1} d r
\end{aligned}
$$

for some points $\theta_{\varepsilon}^{1}=\theta_{\varepsilon}^{1}(r)$ and $\theta_{\varepsilon}^{2}=\theta_{\varepsilon}^{2}(r)$ lying in the intervals whose endpoints are $v^{\prime}(r)$ and $v_{\varepsilon}^{\prime}(r)$ and $v(r)$ and $v_{\varepsilon}(r)$ respectively for a.e. $0<r \leq 1$.

We abbreviate

$$
A_{\varepsilon}(r)=\Phi_{\xi}\left(\theta_{\varepsilon}^{1}, \frac{v_{\varepsilon}}{r}\right) \quad \text { and } \quad B_{\varepsilon}(r)=\Phi_{\eta}\left(v^{\prime}, \frac{\theta_{\varepsilon}^{2}}{r}\right)
$$

for a.e. $r \in(0,1]$ so that the differential quotient of $J_{V}$ becomes

$$
\frac{J_{V}\left(v_{\varepsilon}\right)-J_{V}(v)}{\varepsilon \sigma_{N}}=\int_{0}^{1} A_{\varepsilon}(r) \psi(r) r^{N-1} d r+\int_{0}^{1} B_{\varepsilon}(r)\left(\frac{1}{r} \int_{0}^{r} \psi d \rho\right) r^{N-1} d r
$$

and we note that $A_{\varepsilon}(r) \rightarrow \Phi_{\xi}\left(v^{\prime}, v / r\right)$ and $B_{\varepsilon}(r) \rightarrow \Phi_{\eta}\left(v^{\prime}, v / r\right)$ as $\varepsilon \rightarrow 0$ for a.e. $0<r \leq 1$. 
Now, we show that we can pass to the limit within the integrals in (3.7) by dominated convergence. To this aim, we estimate the functions $A_{\varepsilon}$ and $B_{\varepsilon}$. The functions $A_{\varepsilon} \psi$ appearing in the first integral are different from zero on the set $E_{k}$ only and then, since $\Phi_{\xi}$ is continuous and both $v^{\prime}$ and $v / r$ are bounded over $E_{k}$, it follows that $\left|A_{\varepsilon} \psi\right|$ is bounded as well by a constant depending only on $k$ and $v$ for sufficiently small $|\varepsilon|$.

We now turn to the functions $B_{\varepsilon}$ which, in view of the definition of $\Phi$ in (2.12), we write as the sum of two terms $B_{\epsilon}=B_{\epsilon}^{1}+B_{\epsilon}^{2}$ where $B_{\epsilon}^{i}(r)=\Phi_{\eta}^{i}\left(v^{\prime}, \theta_{\varepsilon}^{2} / r\right)$.

We first estimate $B_{\epsilon}^{1}$ which is multiplied by the integral of $\psi$ over the interval $[0, r]$. As $\psi$ is supported on $E_{k}$, this integral vanishes for $0<r<1 / k$ and the estimate

$$
0<v(1 / k)-\varepsilon_{0}|\psi|_{\infty} \leq \frac{\theta_{\varepsilon}^{2}(r)}{r} \leq k\left(\lambda+\varepsilon_{0}|\psi|_{\infty}\right), \quad 1 / k \leq r \leq 1,
$$

holds for sufficiently small $0<|\varepsilon| \leq \varepsilon_{0}$. Here, $|\psi|_{\infty}$ obviously stands for the $L^{\infty}$ norm of $\psi$. Therefore, we obtain from (2.14) that

$$
\left|B_{\varepsilon}^{1}(r) \frac{1}{r} \int_{0}^{r} \psi d \rho\right| \leq C\left[1+\left(v^{\prime}\right)^{p-1}\right]
$$

holds for a.e. $0<r \leq 1$ for some constant $C=C\left(N, k, \lambda,|\psi|_{\infty}\right)$ and, once multiplied by $r^{N-1}$, the right hand side is integrable over the interval $(0,1]$ because $J$ is finite at $v$.

We then turn to $B_{\epsilon}^{2}$ and we exploit (2.15) with $\xi=v^{\prime}, \eta=v / r$ and $\bar{\eta}=\theta_{\varepsilon}^{2} / r$ for $1 / k \leq r \leq 1$. Then, $\bar{\eta} / \eta=\theta_{\varepsilon}^{2} / v$ and

$$
\left|\frac{\theta_{\varepsilon}^{2}(r)}{v(r)}-1\right| \leq|\varepsilon| \frac{|\psi|_{\infty}}{v(1 / k)}, \quad 1 / k \leq r \leq 1,
$$

so that the ratio $\bar{\eta} / \eta$ is uniformly close to one for $1 / k \leq r \leq 1$ provided $|\varepsilon|$ is small enough. Thus,

$$
\left|B_{\varepsilon}^{2}(r) \frac{1}{r} \int_{0}^{r} \psi d \rho\right| \leq C w\left(v^{\prime}\left(\frac{v}{r}\right)^{N-1}\right)
$$

holds for a.e. $0<r \leq 1$ for some constant $C=C\left(N, k, \lambda,|\psi|_{\infty}\right)$ and again, upon multiplication by $r^{N-1}$, the right hand side is integrable over the interval $(0,1]$.

Therefore, we can pass to the limit in the (rescaled) differential quotient of $J_{V}$ by the dominated convergence theorem and the limit must be zero because of the minimality of $v$. Thus, we obtain

$$
\int_{0}^{1}\left\{\Phi_{\xi}\left(v^{\prime}, \frac{v}{r}\right) r^{N-1} \psi+\Phi_{\eta}\left(v^{\prime}, \frac{v}{r}\right) r^{N-2} \int_{0}^{r} \psi d \rho\right\} d r=0
$$

for every $\psi \in L^{\infty}(0,1)$ such that (3.6) holds.

Integrating by parts in the equation above and recalling again that the integral of $\psi$ over the interval $[0, r]$ vanishes for $0<r<1 / k$, we conclude that the equality

$$
\int_{0}^{1}\left\{\Phi_{\xi}\left(v^{\prime}, \frac{v}{r}\right) r^{N-1}-\int_{1}^{r} \Phi_{\eta}\left(v^{\prime}, \frac{v}{\rho}\right) \rho^{N-2} d \rho\right\} \psi d r=0
$$


holds for every $\psi \in L^{\infty}(0,1)$ satisfying (3.6). Hence,

$$
\Phi_{\xi}\left(v^{\prime}, \frac{v}{r}\right) r^{N-1}-\int_{1}^{r} \Phi_{\eta}\left(v^{\prime}, \frac{v}{\rho}\right) \rho^{N-2} d \rho=c_{k}
$$

for a.e. $r \in E_{k}$ for some constant $c_{k}$. As the sets $E_{k}$ are increasing and their union is the whole interval $(0,1]$, up to a negligible set, we conclude that the constants $c_{k}$ are actually independent from $k$. Thus, we have

$$
\Phi_{\xi}\left(v^{\prime}, \frac{v}{r}\right) r^{N-1}-\int_{1}^{r} \Phi_{\eta}\left(v^{\prime}, \frac{v}{\rho}\right) \rho^{N-2} d \rho=c
$$

for a.e. $0<r \leq 1$. This establishes (b) and (c) follows by differentiation.

Finally, we are left to prove (d). In view of (b), the function $r \mapsto r^{N-1} \Phi_{\xi}\left(v^{\prime}, v / r\right)$ is locally bounded in $(0,1]$. Therefore, since $w^{\prime}(t) \rightarrow-\infty$ as $t \rightarrow 0^{+}$and $\theta^{\prime}(t) \rightarrow+\infty$ as $t \rightarrow+\infty$, we easily obtain $(\mathrm{d})$.

The previous result yields the regularity of minimizers of $J_{V}$ as in Proposition 6.1 in [1].

Corollary 3.4. Assume that $(\mathrm{H} 1), \ldots,(\mathrm{H} 8)$ hold and let $v \in \mathcal{A}(\lambda)$ be a minimizer of $J_{V}$. Then,

(a) $v \in \mathcal{C}^{1}((0,1])$ and $v^{\prime}>0$ on $(0,1]$;

(b) the mapping $r \in(0,1] \mapsto r^{N-2} \Phi_{\eta}\left(v^{\prime}, v / r\right)$ is in $\mathcal{C}((0,1])$;

(c) the mapping $r \in(0,1] \mapsto r^{N-1} \Phi_{\xi}\left(v^{\prime}, v / r\right)$ is in $\mathcal{C}^{1}((0,1])$;

(d) the EL equation (3.4) holds pointwise on $(0,1]$.

Proof. The mapping $\Phi(\xi, \eta)$ for $\xi>0, \eta>0$ is strictly convex in each variable by (H2) and (H5) and

$$
\lim _{\xi \rightarrow 0^{+}} \Phi_{\xi}(\xi, \eta)=-\infty \quad \text { and } \quad \lim _{\xi \rightarrow+\infty} \Phi_{\xi}(\xi, \eta)=+\infty
$$

for every $\eta>0$. Thus, Proposition 6.1 in [1] applies.

Moreover, as $\theta$ and $w$ are of class $C^{2}$ and the second derivative $\Phi_{\xi \xi}$ is positive on $(0,+\infty) \times(0,+\infty)$ because of the convexity of $\theta$ and $w$ and the hypothesis $\theta^{\prime}>0$ on $(0,+\infty)$, it follows that $v$ is actually in $C^{2}((0,1])$ and the EL equation (3.4) turns into

$$
r \Phi_{\xi \xi}\left(v^{\prime}, \frac{v}{r}\right) v^{\prime \prime}=\Phi_{\eta}\left(v^{\prime}, \frac{v}{r}\right)-(N-1) \Phi_{\xi}\left(v^{\prime}, \frac{v}{r}\right)-\Phi_{\eta \xi}\left(v^{\prime}, \frac{v}{r}\right)\left[v^{\prime}-\frac{v}{r}\right] .
$$

So far, we haven't exploited yet the possibility that the minimizers $v \in \mathcal{A}(\lambda)$ of $J_{V}$ be singular at $r=0$, i.e. have $v(0)>0$. This allows for a different choice of the variations $v_{\varepsilon}$ in the proof of Theorem 3.3, thus letting the volume part of $J_{V}$ come into play.

Theorem 3.5. Assume that $(\mathrm{H} 1), \ldots,(\mathrm{H} 8)$ hold and let $v \in \mathcal{A}(\lambda)$ be a minimizer of $J_{V}$ such that $v(0)>0$. Then, 
(a) the mapping $r \in(0,1] \mapsto r^{N-2} \Phi_{\eta}\left(v^{\prime}, v / r\right)$ is in $L^{1}(0,1)$;

(b) the mapping $r \in(0,1] \mapsto r^{N-1} \Phi_{\xi}\left(v^{\prime}, v / r\right)$ is in $A C([0,1])$; and, setting

$$
T(r)=\left[\frac{r}{v(r)}\right]^{N-1} \Phi_{\xi}\left(v^{\prime}(r), v(r) / r\right), \quad 0<r \leq 1,
$$

we have

(c) $\lim _{r \rightarrow 0^{+}} T(r)=w^{\infty}$.

The limit of $T$ as $r \rightarrow 0^{+}$is the radial component of the Cauchy stress tensor on the boundary of the cavity.

Proof. Statement (a) follows from (3.5) as $r_{0} \rightarrow 0^{+}$and (b) follows immediately from this and (3.8).

To prove (c), consider the variations $v_{\varepsilon}=v+\varepsilon \varphi$ where $\varphi \in C^{\infty}([0,1])$ is such that $0 \leq \varphi \leq 1, \varphi=1$ on the interval $\left[0, r_{0}\right]$ for some $0<r_{0}<1$ and $\varphi(1)=0$. Since $v(0)>0, v_{\varepsilon}$ is in $\mathcal{A}(\lambda)$ for every sufficiently small $\varepsilon \neq 0$ by (a) of Corollary 3.4. Now, as in Theorem 3.3, we compute the (rescaled) differential quotient of $J_{V}$ at $v$ with increment $v_{\varepsilon}-v$. In view of the equality $v_{\varepsilon}(0)=v(0)+\varepsilon$ and the mean value theorem, we have

$$
\frac{J_{V}\left(v_{\varepsilon}\right)-J_{V}(v)}{\varepsilon \sigma_{N}}=\int_{0}^{1}\left[A_{\varepsilon} \varphi^{\prime}+B_{\varepsilon} \frac{\varphi}{r}\right] r^{N-1} d r+\frac{w^{\infty}}{N} \frac{[v(0)+\varepsilon]^{N}-[v(0)]^{N}}{\varepsilon}
$$

for every sufficiently small $\varepsilon \neq 0$ where $A_{\varepsilon}$ and $B_{\varepsilon}$ are defined as in the proof of Theorem 3.3 for some points $\theta_{\varepsilon}^{1}=\theta_{\varepsilon}^{1}(r)$ and $\theta_{\varepsilon}^{2}=\theta_{\varepsilon}^{2}(r)$ lying in the intervals whose endpoints are $v^{\prime}(r)$ and $v_{\varepsilon}^{\prime}(r)$ and $v(r)$ and $v_{\varepsilon}(r)$ respectively for every $0<r \leq 1$.

We want to prove that we can pass to the limit in the integral above. To this aim, we write the integral over $(0,1]$ as the the sum of the integrals over $\left(0, r_{0}\right]$ and $\left[r_{0}, 1\right]$. As $v$ and $v_{\varepsilon}$ are smooth on $(0,1]$ by Corollary 3.4 , it is clear that all the functions $v_{\varepsilon} / r, \theta_{\varepsilon}^{2} / r, \theta_{\varepsilon}^{1}$ and $v^{\prime}$ remain in a compact subset of $(0, \infty)$ as $r$ ranges between $r_{0}$ and 1 . As $\Phi_{\xi}$ and $\Phi_{\eta}$ are continuous on the same set, the functions $A_{\varepsilon}$ and $B_{\varepsilon}$ are bounded over the interval $\left[r_{0}, 1\right]$ uniformly with respect to small $\varepsilon \neq 0$ and we can pass to the limit in the integral above over the interval $\left[r_{0}, 1\right]$.

As to the integral over $\left(0, r_{0}\right]$, we have $\varphi=1$ and $\varphi^{\prime}=0$ on $\left(0, r_{0}\right]$ and hence it reduces to

$\int_{0}^{r_{0}}\left[A_{\varepsilon} \varphi^{\prime}+B_{\varepsilon} \frac{\varphi}{r}\right] r^{N-1} d r=\int_{0}^{r_{0}} \Phi_{\eta}\left(v^{\prime}, \frac{\theta_{\varepsilon}^{2}}{r}\right) r^{N-2} d r=\int_{0}^{r_{0}}\left[B_{\varepsilon}^{1}+B_{\varepsilon}^{2}\right] r^{N-2} d r$

where $B_{\varepsilon}^{1}$ and $B_{\varepsilon}^{2}$ are defined as in the proof of Theorem 3.3 and can be estimated by similar arguments.

Infact, recalling that $\theta_{\varepsilon}^{2}$ lies between $v$ and $v_{\varepsilon}$ on $[0,1]$ and that $\left|v_{\varepsilon}-v\right| \leq \varepsilon$ on the same interval, we have that $\left|\theta_{\varepsilon}^{2} / v-1\right| \leq|\varepsilon| / v(0)$ and $v_{\varepsilon}>v(0) / 2>0$ hold on $[0,1]$ for $|\varepsilon|$ small enough. Hence, from (2.13) and (H3), we obtain for $0<r \leq 1$ 


$$
\left|B_{\varepsilon}^{1}\right| r^{N-2} \leq C\left[1+\frac{1}{\theta_{\varepsilon}^{2}} \Phi^{1}\left(v^{\prime}, \frac{\theta_{\varepsilon}^{2}}{r}\right) r^{N-1}\right] \leq C\left[1+\frac{1}{v(0)} \Phi^{1}\left(v^{\prime}, \frac{v}{r}\right) r^{N-1}\right]
$$

which is obviously integrable on the same interval.

Finally, recalling again that $\theta_{\varepsilon}^{2} / v \rightarrow 1$ uniformly on $[0,1]$ as $\varepsilon \rightarrow 0$, from (2.15) we obtain for $B_{\varepsilon}^{2}$ that

$$
\left|B_{\varepsilon}^{2}\right| r^{N-2} \leq C \frac{1}{v} \Phi^{2}\left(v^{\prime}, \frac{v}{r}\right) r^{N-1} \leq \frac{C}{v(0)} w\left(v^{\prime}\left(\frac{v}{r}\right)^{N-1}\right) r^{N-1}
$$

and again the right hand side is integrable over $(0,1]$ because $J_{V}(v)<\infty$.

Therefore, we can pass to the limit in (3.10) and the limit must vanish because $v$ is a minimizer. Thus,

$$
\int_{0}^{1}\left[\Phi_{\xi}\left(v^{\prime}, \frac{v}{r}\right) \varphi^{\prime}+\Phi_{\eta}\left(v^{\prime}, \frac{v}{r}\right) \frac{\varphi}{r}\right] r^{N-1} d r+w^{\infty}[v(0)]^{N-1}=0 .
$$

Integrating by parts and recalling that $\varphi(0)=1$ and $\varphi(1)=0$, we obtain

$$
\begin{aligned}
w^{\infty}[v(0)]^{N-1}= & \lim _{r \rightarrow 0}\left[\Phi_{\xi}\left(v^{\prime}, \frac{v}{r}\right) r^{N-1}\right] \\
& +\int_{0}^{1}\left\{\frac{\mathrm{d}}{\mathrm{d} r}\left[\Phi_{\xi}\left(v^{\prime}, \frac{v}{r}\right) r^{N-1}\right]-\Phi_{\eta}\left(v^{\prime}, \frac{v}{r}\right) r^{N-2}\right\} \varphi d r
\end{aligned}
$$

and hence (c) follows from (3.4).

The analysis developed so far thus shows that, whenever $(\mathrm{H} 1), \ldots,(\mathrm{H} 8)$ hold, the optimality conditions for minimizers of $J_{V}$ are the EL equation (3.4) which we rewrite as

$$
r \frac{\mathrm{d}}{\mathrm{d} r}\left[\Phi_{\xi}\left(v^{\prime}, \frac{v}{r}\right)\right]=\Phi_{\eta}\left(v^{\prime}, \frac{v}{r}\right)-(N-1) \Phi_{\xi}\left(v^{\prime}, \frac{v}{r}\right), \quad 0<r \leq 1,
$$

together with the boundary conditions

$$
\left\{\begin{array} { l } 
{ v ( 0 ) = 0 } \\
{ v ( 1 ) = \lambda }
\end{array} \text { or } \quad \left\{\begin{array}{l}
v(0)>0 \text { and } \lim _{r \rightarrow 0_{+}} T(r)=w^{\infty} \\
v(1)=\lambda
\end{array}\right.\right.
$$


and that every solution to (3.4), regardless of the boundary conditions, is (absolutely) continuous on $[0,1]$ and continuously differentiable on $(0,1]$.

The shooting method. Following Stuart's ideas in [10], we can investigate the properties of possible solutions to the EL equation (3.4), (3.11) by looking at the solutions to the backward Cauchy problem

$$
\left\{\begin{array}{l}
r \frac{\mathrm{d}}{\mathrm{d} r}\left[\Phi_{\xi}\left(v^{\prime}, \frac{v}{r}\right)\right]=\Phi_{\eta}\left(v^{\prime}, \frac{v}{r}\right)-(N-1) \Phi_{\xi}\left(v^{\prime}, \frac{v}{r}\right) \quad \text { for } 0<r \leq 1 \\
v(1)=\lambda \text { and } v^{\prime}(1)=\alpha
\end{array}\right.
$$

with initial data $\alpha>0$ and $\lambda>0$. We remark here that, for future purposes, it is convenient to consider the Cauchy problem (3.13) not only for $\lambda \geq 1$ but also for $\lambda>0$. Because of the remark following Corollary 3.4, for every choice of the initial data this problem has a unique maximal solution $v_{\alpha} \in C^{2}\left(I_{\alpha}\right)$ where $I_{\alpha} \subset(0,+\infty)$. In particular, $v_{\lambda}(r)=\lambda r$ for $r>0$ is the maximal solution to (3.13) corresponding to $\alpha=\lambda$. Though our hypothesis on $\Phi$ are not the same as those in [10], Stuart's arguments can be easily adapted to the case considered here and we summarize the properties of $v_{\alpha}$ in the following lemma which corresponds to Lemma 1 and Lemma 2 in [10].

Lemma 3.6. Let $v_{\alpha} \in C^{2}\left(I_{\alpha}\right)$ be the maximal solution to (3.13) corresponding to $\lambda>0$ and $\alpha>0$. Then,

(a) $\inf I_{\alpha}>1-\lambda / \alpha>0$ for $\alpha>\lambda$;

(b) $\inf I_{\alpha}=0$ for $0<\alpha \leq \lambda$.

Moreover, for every $0<\alpha<\lambda, v_{\alpha}$ has the following properties for every $0<r \leq 1$ :

(c) $v_{\alpha}^{\prime \prime}(r)>0$;

(d) $0<v_{\alpha}^{\prime}(r)<\alpha$;

(e) $0<\lambda-\alpha<v_{\alpha}(r)<\lambda$;

(f) $\frac{\mathrm{d}}{\mathrm{d} r}\left(\frac{v_{\alpha}(r)}{r}\right)<0$.

This shows in particular that the only nonsingular solution to the EL equation $(3.11),(3.12)$ for $J_{V}$ is the linear solution $v_{\lambda}$.

We finally show that for every $\lambda \geq 1, v_{\lambda}$ is the unique solution to the EL equation (3.11), (3.12) for $J_{V}$ and therefore the unique minimizer of $J_{V}$.

Indeed, by the previous analysis, it is enough to prove that no solution $v=v_{\alpha}$ to (3.13) with $0<\alpha<\lambda$ can take the boundary condition $T_{\alpha}(r) \rightarrow w^{\infty}$ as $r \rightarrow 0_{+}$and have finite energy $J_{V}(v)<+\infty$ at the same time. This follows from the following energy estimate (see Lemma 9 in [10]).

Assume that a solution $v$ to $(3.11),(3.12)$ with $v(0)>0$ exists and that it has finite energy, i.e. $J_{V}(v)<+\infty$. Then, $v=v_{\alpha}$ for some $0<\alpha<\lambda$. As everything is smooth, an easy computation (see eq. (6.12) in [1]) shows that the equality

$$
\frac{\mathrm{d}}{\mathrm{d} r}\left\{r^{N}\left[\Phi\left(v^{\prime}, \frac{v}{r}\right)-\left(v^{\prime}-\frac{v}{r}\right) \Phi_{\xi}\left(v^{\prime}, \frac{v}{r}\right)\right]\right\}=N r^{N-1} \Phi\left(v^{\prime}, \frac{v}{r}\right)
$$


holds for every $0<r \leq 1$. Integrating between $\varepsilon$ and 1 we obtain

$$
\begin{aligned}
& N \int_{\varepsilon}^{1} \Phi\left(v^{\prime}, \frac{v}{r}\right) r^{N-1} d r=\left[\Phi(\alpha, \lambda)+(\lambda-\alpha) \Phi_{\xi}(\alpha, \lambda)\right] \\
& \quad-\varepsilon^{N}\left\{\Phi\left(v^{\prime}(\varepsilon), \frac{v(\varepsilon)}{\varepsilon}\right)-\left(v^{\prime}(\varepsilon)-\frac{v(\varepsilon)}{\varepsilon}\right) \Phi_{\xi}\left(v^{\prime}(\varepsilon), \frac{v(\varepsilon)}{\varepsilon}\right)\right\}
\end{aligned}
$$

and hence

$$
\begin{aligned}
& N \int_{\varepsilon}^{1} \Phi\left(v^{\prime}, \frac{v}{r}\right) r^{N-1} d r+[v(\varepsilon)]^{N}\left[1-\varepsilon \frac{v^{\prime}(\varepsilon)}{v(\varepsilon)}\right] T(\varepsilon) \\
& +\varepsilon^{N} \Phi\left(v^{\prime}(\varepsilon), \frac{v(\varepsilon)}{\varepsilon}\right)=\left[\Phi(\alpha, \lambda)+(\lambda-\alpha) \Phi_{\xi}(\alpha, \lambda)\right] .
\end{aligned}
$$

Now, $T(\varepsilon) \rightarrow w^{\infty}$ as $\varepsilon \rightarrow 0^{+}$by (3.12) and $\varepsilon v^{\prime}(\varepsilon) \rightarrow 0$ by (d) of Lemma 3.6. As $J_{V}(v)<+\infty$, we conclude that

$$
\lim _{\varepsilon \rightarrow 0^{+}} \varepsilon^{N} \Phi\left(v^{\prime}(\varepsilon), \frac{v(\varepsilon)}{\varepsilon}\right)=0,
$$

otherwise it would be $J_{V}(v)=+\infty$. Thus, for a singular solution $v=v_{\alpha}$ of (3.13) with finite energy, we would have

$$
J_{V}(v)=\frac{\sigma_{N}}{N}\left[\Phi(\alpha, \lambda)+(\lambda-\alpha) \Phi_{\xi}(\alpha, \lambda)\right]<\frac{\sigma_{N}}{N} \Phi(\lambda, \lambda)=J_{V}\left(v_{\lambda}\right)
$$

by the strict convexity of $\xi \in(0,+\infty) \mapsto \Phi(\xi, \eta)$ which follows from (H2) and (H5). We have thus proved the following result:

Theorem 3.7. Assume that $(\mathrm{H} 1), \ldots$, (H8) hold. Then, $v_{\lambda}$ is the unique minimizer of $J_{V}$ on $\mathcal{A}(\lambda)$ for every $\lambda \geq 1$.

\section{The surface model}

In this section, we consider the model where the energy associated with a radially symmetric deformation $v \in \mathcal{A}(\lambda)$ is given by

$$
J_{S}(v)=J(v)+w^{\infty} \sigma_{N}[v(0)]^{N-1}, \quad v \in \mathcal{A}(\lambda),
$$

and $J$ is defined by (2.17) as before. This is the energy studied by Müller and Spector in [7], here considered in the very simplified situation of radially symmetric deformations. Note however that, following Blatz and Ko experiments in [2] and contrary to [7], we assume (H8), i.e. the energy density of $J$ has linear growth with respect to the Jacobian determinant.

For this model, we want to prove that the energy $J_{S}$ has a singular minimizer $v$ with $v(0)>0$ for large enough $\lambda>1$.

The first issue we have to set is the existence of minimizers for $J_{S}$. Indeed, the definition of $J_{V}$ and the integral representation formula given by Theorem 3.1 show that $J_{S}$ cannot be lower semicontinuous with respect to the weak convergence (2.18) at any function $v \in \mathcal{A}(\lambda)$ such that $0<v(0)<N$ because $J_{S}(v)>J_{V}(v)$ at any such $v$. Thus, the existence of minimizers for $J_{S}$ does not follow straightforwardly from direct methods. Yet, attainment for $J_{S}$ can be proved by showing that $J_{S}$ is actually lower semicontinuous along minimizing 
sequences. Instead of going this way, we shall go through relaxation, i.e. we are going to extend $J_{S}$ as a lower semicontinuous functional, say $J_{\Gamma}$, defined on a larger class of objects and show that $J_{\Gamma}$ attains its minimum on this larger class and that the minimum is actually achieved at a function $v$ from $\mathcal{A}(\lambda)$.

The underlying idea is that for the weak convergence $v_{k} \rightarrow v$ considered in (2.18), while $v_{k} \rightarrow v$ pointwise in $(0,1]$, it may happen that the values $v_{k}(0)$ of the approximating functions at $r=0$ converge to a value, say $\varepsilon \geq 0$, strictly smaller than $v(0)$, i.e. $0 \leq \varepsilon \leq v(0)$. Thus, if we look at the graphs of the functions, what the sequence $\left\{v_{k}\right\}$ is really approximating is the graph of $v$ and a vertical part over the origin, the segment $[\varepsilon, v(0)]$ and, if we want to extend the definition of $J_{S}$ by lower semicontinuity, we have to keep track of this vertical part of the graph.

This suggests we consider the set

$$
\Gamma=\{(v, \varepsilon): v \in \mathcal{A}(\lambda) \text { and } 0 \leq \varepsilon \leq v(0)\}
$$

of "graphs" which are (weak) limits of "regular graphs", i.e. graphs associated to functions $v \in \mathcal{A}(\lambda)$. We denote the subset of regular graphs by

$$
\Gamma_{0}=\{(v, \varepsilon) \in \Gamma: v(0)=\varepsilon\}
$$

and we endow $\Gamma$ with the convergence $\left(v_{k}, \varepsilon_{k}\right) \rightarrow(v, \varepsilon)$ given by $v_{k} \rightarrow v$ weakly in $\mathcal{A}(\lambda)$ and $\varepsilon_{k} \rightarrow \varepsilon$. It is easy to check that $\Gamma$ is closed with respect to this convergence. The functional $J_{S}$ is actually defined on regular graphs by

$$
J_{S}(v, \varepsilon)=J(v)+w^{\infty} \sigma_{N} \varepsilon^{N-1}, \quad(v, \varepsilon) \in \Gamma_{0},
$$

and we consider its lower semicontinuous extension $J_{\Gamma}$ defined on $\Gamma$ by

$$
J_{\Gamma}(v, \varepsilon)=\inf \left\{\liminf _{k} J_{S}\left(v_{k}, \varepsilon_{k}\right):\left(v_{k}, \varepsilon_{k}\right) \in \Gamma_{0} \text { and }\left(v_{k}, \varepsilon_{k}\right) \rightarrow(v, \varepsilon)\right\}
$$

for every $(v, \varepsilon) \in \Gamma$. This construction can be described in the language of currents, see Section 2.6.3 in [4].

The claim that $J_{S}$ has singular minimizers for large enough $\lambda>1$ follows from the following steps.

Integral representation for $\boldsymbol{J}_{\boldsymbol{\Gamma}}$. By the same arguments of Marcellini's relaxation result (Theorem 1 in [6]), we prove the following representation formula for $J_{\Gamma}$.

Theorem 4.1. Assume that (H1), (H2), (H3), (H4) and (H5) hold. Then,

$$
J_{\Gamma}(v, \varepsilon)=J(v)+w^{\infty} \frac{\sigma_{N}}{N}\left\{[v(0)]^{N}-\varepsilon^{N}\right\}+w^{\infty} \sigma_{N} \varepsilon^{N-1}, \quad(v, \varepsilon) \in \Gamma .
$$

The meaning of the additional terms in $J_{\Gamma}$ is transparent.

Proof. Let $\bar{J}$ be the right hand side of (4.1). We prove that $J_{\Gamma} \geq \bar{J}$, i.e. that

$$
\liminf _{k} J_{S}\left(v_{k}, \varepsilon_{k}\right) \geq \bar{J}(v, \varepsilon)
$$


holds for every $(v, \varepsilon) \in \Gamma$ and every sequence of regular graphs $\left(v_{k}, \varepsilon_{k}\right) \in \Gamma_{0}$ such that $v_{k} \rightarrow v$ weakly in $\mathcal{A}(\lambda)$ and $\varepsilon_{k} \rightarrow \varepsilon$. Assuming without loss of generality that

$$
\liminf _{k} J_{S}\left(v_{k}, \varepsilon_{k}\right)=\lim _{k} J_{S}\left(v_{k}, \varepsilon_{k}\right),
$$

there are two possibilities: either $v(0)=\varepsilon \geq 0$ or $v(0)>\varepsilon \geq 0$.

In the first case, we have $\bar{J}(v, \varepsilon)=J_{S}(v, \varepsilon)$ and hence

$$
\begin{aligned}
\liminf _{k} J_{S}\left(v_{k}, \varepsilon_{k}\right) & \geq \liminf _{k} J\left(v_{k}\right)+w^{\infty} \sigma_{N} \varepsilon^{N-1} \\
& \geq J(v)+w^{\infty} \sigma_{N} \varepsilon^{N-1}=J_{S}(v, \varepsilon)=\bar{J}(v, \varepsilon)
\end{aligned}
$$

because $J$ is sequentially lower semicontinuous with respect to the weak convergence $v_{k} \rightarrow v$. If the other case $v(0)>\varepsilon \geq 0$ occurs, recalling that $v$ is strictly increasing and that $v_{k} \rightarrow v$ pointwise on $(0,1]$, we find integers $k_{n+1}>k_{n}$ such that $v_{k_{n}}(1 / n)>v(0)$. Set

$$
\bar{v}_{n}(\rho)=n[v(0)-\varepsilon] \rho+\varepsilon, \quad 0 \leq \rho \leq 1
$$

and note that $\bar{v}_{n}(1 / n)=v(0)<v_{k_{n}}(1 / n)$. Since $\bar{v}_{n}(1) \rightarrow+\infty$ as $n \rightarrow+\infty$ and $v_{k_{n}}(1)=\lambda$ for every $n$, we find $1 / n<\rho_{n}<1$ such that the equality

$$
v_{k_{n}}\left(\rho_{n}\right)=\bar{v}_{n}\left(\rho_{n}\right)=n[v(0)-\varepsilon] \rho_{n}+\varepsilon
$$

holds for large enough $n$ and moreover

$$
0<\frac{1}{n}<\rho_{n}=\frac{v_{k_{n}}\left(\rho_{n}\right)-\varepsilon}{n[v(0)-\varepsilon]}<\frac{1}{n} \cdot \frac{\lambda-\varepsilon}{v(0)-\varepsilon} \rightarrow 0 .
$$

Then, we estimate the limit of $J_{S}$ along the sequence $\left(v_{k}, \varepsilon_{k}\right)$. Since $\Phi^{1} \geq 0$, we have

$$
\begin{aligned}
\lim _{k} J_{S}\left(v_{k}, \varepsilon_{k}\right) \geq & \liminf _{n} \sigma_{N} \int_{0}^{\rho_{n}} w\left(v_{k_{n}}^{\prime}\left(\frac{v_{k_{n}}}{r}\right)^{N-1}\right) r^{N-1} d r \\
& +\liminf _{n} \sigma_{N} \int_{\rho_{n}}^{1}\left[\Phi^{1}\left(v_{k_{n}}^{\prime}, \frac{v_{k_{n}}}{r}\right)+w\left(v_{k_{n}}^{\prime}\left(\frac{v_{k_{n}}}{r}\right)^{N-1}\right)\right] r^{N-1} d r \\
& +w^{\infty} \sigma_{N} \varepsilon^{N-1} \\
= & A+B+w^{\infty} \sigma_{N} \varepsilon^{N-1}
\end{aligned}
$$

and we claim that

$$
A \geq w^{\infty} \frac{\sigma_{N}}{N}\left\{[v(0)]^{N}-\varepsilon^{N}\right\} \quad \text { and } \quad B \geq J(v)
$$

which all together yield (4.2).

We consider the term $B$ first. For every $0<\eta<1$, we have

$$
\begin{aligned}
& \sigma_{N} \int_{\rho_{n}}^{1}\left[\Phi^{1}\left(v_{k_{n}}^{\prime}, \frac{v_{k_{n}}}{r}\right)+w\left(v_{k_{n}}^{\prime}\left(\frac{v_{k_{n}}}{r}\right)^{N-1}\right)\right] r^{N-1} d r \\
& \quad \geq \sigma_{N} \int_{\eta}^{1}\left[\Phi^{1}\left(v_{k_{n}}^{\prime}, \frac{v_{k_{n}}}{r}\right)+w\left(v_{k_{n}}^{\prime}\left(\frac{v_{k_{n}}}{r}\right)^{N-1}\right)\right] r^{N-1} d r
\end{aligned}
$$


since $0<\rho_{n}<\eta$ eventually and the inequality $B \geq J(v)$ then follows by lower semicontinuity letting first $n \rightarrow+\infty$ and then $\eta \rightarrow 0^{+}$. To estimate $A$, we exploit the convexity of $w$ [hypothesis (H5)] and Jensen's inequality. In fact, from

$\int_{0}^{\rho_{n}} r^{N-1} d r=\frac{\rho_{n}^{N}}{N}$ and $\int_{0}^{\rho_{n}} v_{k_{n}}^{\prime}\left(\frac{v_{k_{n}}}{r}\right)^{N-1} r^{N-1} d r=\frac{\left[v_{k_{n}}\left(\rho_{n}\right)\right]^{N}-\varepsilon_{k_{n}}^{N}}{N}$,

and Jensen's inequality, we find

$$
\sigma_{N} \int_{0}^{\rho_{n}} w\left(v_{k_{n}}^{\prime}\left(\frac{v_{k_{n}}}{r}\right)^{N-1}\right) r^{N-1} d r \geq \sigma_{N} \frac{\rho_{n}^{N}}{N} w\left(\frac{\left[v_{k_{n}}\left(\rho_{n}\right)\right]^{N}-\varepsilon_{k_{n}}^{N}}{\rho_{n}^{N}}\right)
$$

and we note that

$$
\lim _{n \rightarrow+\infty} \frac{\left[v_{k_{n}}\left(\rho_{n}\right)\right]^{N}-\varepsilon_{k_{n}}^{N}}{\rho_{n}^{N}}=+\infty
$$

because $\lim _{n} \rho_{n}=0^{+}$and $\liminf _{n} v_{k_{n}}\left(\rho_{n}\right) \geq \liminf _{n} v_{k_{n}}(1 / n) \geq v(0)>\varepsilon=\lim _{n} \varepsilon_{k_{n}}$. Thus,

$$
\begin{aligned}
A & =\liminf _{n} \sigma_{N} \int_{0}^{\rho_{n}} w\left(v_{k_{n}}^{\prime}\left(\frac{v_{k_{n}}}{r}\right)^{N-1}\right) r^{N-1} d r \\
& \geq \lim _{n} \inf \frac{\sigma_{N}}{N}\left(\left[v_{k_{n}}\left(\rho_{n}\right)\right]^{N}-\varepsilon_{k_{n}}^{N}\right) \frac{w\left(\frac{\left[v_{k_{n}}\left(\rho_{n}\right)\right]^{N}-\varepsilon_{k_{n}}^{N}}{\rho_{n}^{N}}\right)}{\frac{\left[v_{k_{n}}\left(\rho_{n}\right)\right]^{N}-\varepsilon_{k_{n}}^{N}}{\rho_{n}^{N}}} \\
& \geq w^{\infty} \frac{\sigma_{N}}{N}\left([v(0)]^{N}-\varepsilon^{N}\right)
\end{aligned}
$$

and this completes the proof of (4.2).

We now pass to the reverse inequality $J_{\Gamma} \leq \bar{J}$ which we prove by exhibiting, for every graph $(v, \varepsilon) \in \Gamma$, a sequence of regular graphs $\left(v_{k}, \varepsilon\right) \in \Gamma_{0}$ such that $v_{k} \rightarrow v$ weakly in $\mathcal{A}(\lambda)$ and

$$
\liminf _{k} J_{S}\left(v_{k}, \varepsilon\right) \leq \bar{J}(v, \varepsilon) .
$$

We can assume that $v(0)>\varepsilon \geq 0$ otherwise the conclusion is obvious (take $v_{k}=v$ for every $k$ ). For large enough $k$, we choose $0<\rho_{k}<1$ such that $k \rho_{k}+\varepsilon=v\left(\rho_{k}\right)$ and we set

$$
v_{k}(\rho)= \begin{cases}k \rho+\varepsilon & 0 \leq \rho \leq \rho_{k} \\ v(\rho) & \rho_{k} \leq \rho \leq 1\end{cases}
$$

It is clear that $\left(v_{k}, \varepsilon\right) \in \Gamma_{0}$ and $v_{k} \rightarrow v$ weakly in $\mathcal{A}(\lambda)$ because $\rho_{k} \rightarrow 0^{+}$. Then,

$$
\begin{aligned}
J_{S}\left(v_{k}, \varepsilon\right) & \leq \sigma_{N} \int_{0}^{\rho_{k}}\left[\Phi^{1}\left(k, k+\frac{\varepsilon}{r}\right)+w\left(k\left(k+\frac{\varepsilon}{r}\right)^{N-1}\right)\right] r^{N-1} d r+J_{S}(v, \varepsilon) \\
& =A_{k}+J_{S}(v, \varepsilon)
\end{aligned}
$$


and, recalling the definition of $\Phi$ in (2.12) as the sum of $\Phi^{1}$ and $\Phi^{2}$, we write $A_{k}$ as $A_{k}=A_{k}^{1}+A_{k}^{2}$ where

$$
A_{k}^{i}=\sigma_{N} \int_{0}^{\rho_{k}} \Phi^{i}\left(k, k+\frac{\varepsilon}{r}\right) r^{N-1} d r \quad i=1,2 .
$$

We claim that

$$
\begin{aligned}
\lim _{k} A_{k}^{1} & =0 \\
\limsup _{k} A_{k}^{2} & \leq w^{\infty} \frac{\sigma_{N}}{N}\left([v(0)]^{N}-\varepsilon^{N}\right) ;
\end{aligned}
$$

whence the conclusion $J_{\Gamma}(v, \varepsilon) \leq \bar{J}(v, \varepsilon)$ follows.

First we prove (4.3). The growth hypothesis (H3) (or the corresponding property $\left(\mathrm{H} 3^{\prime}\right)$ for $\left.\Phi^{1}\right)$ yields

$$
\Phi^{1}\left(k, k+\frac{\varepsilon}{\rho}\right) \leq C\left(1+k^{p}+\frac{\varepsilon^{p}}{\rho^{p}}\right)
$$

for every $k$ and $\rho$ whence, letting $C=C(N, p)$ be a constant that may change from line to line, we obtain

$$
0 \leq A_{k}^{1} \leq C\left[\left(1+k^{p}\right) \rho_{k}^{N}+\varepsilon^{p} \rho_{k}^{N-p}\right]=C\left\{\rho_{k}^{N}+\left[\left(k \rho_{k}\right)^{p}+\varepsilon^{p}\right] \rho_{k}^{N-p}\right\} .
$$

Since $\rho_{k} \rightarrow 0$ and $k \rho_{k}=v\left(\rho_{k}\right)-\varepsilon \rightarrow v(0)-\varepsilon$ as $k \rightarrow+\infty$, (4.3) follows.

As regards (4.4), set

$$
\bar{w}(t)=\frac{w(t)}{w^{\infty} t}-1, \quad t>0,
$$

so that $w(t)=w^{\infty} t[1+\bar{w}(t)]$ for $t>0$ and $\bar{w}(t) \rightarrow 0$ as $t \rightarrow+\infty$. Therefore, given $\eta>0$, we choose $t_{0}=t_{0}(\eta)>0$ such that $|\bar{w}(t)| \leq \eta$ for $t \geq t_{0}$, so that

$$
\left|\bar{w}\left(k\left(k+\frac{\varepsilon}{\rho}\right)^{N-1}\right)\right| \leq \eta, \quad k \geq \sqrt[N]{t_{0}} .
$$
Then, we consider the locally Lipschitz function $u_{k}(x)=v_{k}(|x|) \frac{x}{|x|}$ defined for
$0<|x| \leq \rho_{k}$, and we note that

$$
\operatorname{det} D u_{k}(x)=k\left(k+\frac{\varepsilon}{|x|}\right)^{N-1}, \quad 0<|x| \leq \rho_{k} .
$$

Thus,

$\sigma_{N} \int_{0}^{\rho_{k}} k\left(k+\frac{\varepsilon}{r}\right)^{N-1} r^{N-1} d r=\int_{B_{\rho_{k}}} \operatorname{det} D u_{k}(x) d x=\frac{\sigma_{N}}{N}\left(\left[v\left(\rho_{k}\right)\right]^{N}-\varepsilon^{N}\right)$

and hence, for $k \geq \sqrt[N]{t_{0}}$, we obtain

$$
\begin{aligned}
0 \leq A_{k}^{2} & =\sigma_{N} \int_{0}^{\rho_{k}} w^{\infty} k\left(k+\frac{\varepsilon}{r}\right)^{N-1}\left\{1+\bar{w}\left(k\left(k+\frac{\varepsilon}{r}\right)^{N-1}\right)\right\} r^{N-1} d r \\
& \leq w^{\infty}(1+\eta) \frac{\sigma_{N}}{N}\left(\left[v\left(\rho_{k}\right)\right]^{N}-\varepsilon^{N}\right)
\end{aligned}
$$

whence (4.4) follows. 
Existence and regularity of minimizing graphs. The existence of minimizers of $J_{\Gamma}$ on $\Gamma$ follows straightforwardly from direct methods. In fact, $J_{\Gamma}$ is sequentially lower semicontinuous along weakly converging sequences of graphs in $\Gamma$ by construction and its sublevel sets are closed and sequentially compact for the weak convergence of graphs because the sublevel sets of $J$ enjoy the same properties with respect to the weak convergence in $\mathcal{A}(\lambda)$.

Now, we want to prove that every minimizer $v$ of $J_{\Gamma}$ is a regular graph, i.e. $v(0)=\varepsilon$ whenever $(v, \varepsilon)$ is a minimizer of $J_{\Gamma}$ and that $v$ is a minimizer of $J_{S}$ on $\mathcal{A}(\lambda)$. This can be proved by examining again the EL equation for $J_{V}$.

In fact, let $(v, \varepsilon) \in \Gamma$ be a minimizer of $J_{\Gamma}$ and let play again with variations of the form $\left(v_{t}, \varepsilon\right), t \neq 0$, i.e. we do not make any variation in the $\varepsilon$ direction. We first choose $v_{t}$ to be the very same variations of the proof of Theorem 3.3, that is

$$
v_{t}(r)=v(r)+t \int_{0}^{r} \psi d \rho, \quad 0<r \leq 1,
$$

where $\psi$ satisfies (3.6) and the sets $E_{k}$ are those defined in the same theorem. Thus

$$
\frac{J_{\Gamma}\left(v_{t}, \varepsilon\right)-J_{\Gamma}(v, \varepsilon)}{t}=\frac{J_{V}\left(v_{t}\right)-J_{V}(v)}{t}
$$

and therefore all the conclusions of Theorem 3.3 and Corollary 3.4 remain true for $v$ as well. In particular, $v \in \mathcal{C}^{2}((0,1])$ is a solution to the EL equation (3.4). If it happened that the minimizer $(v, \varepsilon)$ were not a regular graph, i.e. $v(0)>\varepsilon \geq 0$, we could then choose functions $v_{t}=v+t \varphi$ with $\varphi(0)=1$ as in the proof of Theorem 3.5 and the resulting pairs $\left(v_{t}, \varepsilon\right)$ would be admissible variations for $J_{\Gamma}$ for small $t \neq 0$. Once more, as we do not make any variations in the $\varepsilon$ direction, $v$ would be a solution to the EL equation (3.11) for $J_{V}$ with the boundary condition (3.12) corresponding to $v(0)>0$, which we know does not exist. Thus, $v(0)=\varepsilon$ and it is then obvious that $v$ is also a minimizer of $J_{S}$ on $\mathcal{A}(\lambda)$. We remark that, whenever the pair $(v, \varepsilon)$ is a regular graph, the last part of the previous argument breaks down because the variations $\left(v_{t}, \varepsilon\right)$ are never admissible for $t<0$. We have thus proved the following result.

Theorem 4.2. Assume that $(\mathrm{H} 1), \ldots,(\mathrm{H} 8)$ hold. For every $\lambda>1$, there exists a minimizer $(v, \varepsilon) \in \Gamma$ of $J_{\Gamma}$ and every minimizer $(v, \varepsilon) \in \Gamma$ of $J_{\Gamma}$ has the following properties:

(a) $v(0)=\varepsilon$;

(b) $v$ is a minimizer of $J_{S}$ on $\mathcal{A}(\lambda)$.

Existence of singular minimizer of $\boldsymbol{J}_{\boldsymbol{S}}$. We first note that the linear function $v_{\lambda}$ is not a minimizer of $J_{S}$ for $\lambda \gg 1$. This follows from the very same argument of Proposition 7.6 in [1]. Set

$$
\bar{v}_{\lambda}(r)=\frac{\lambda}{\sqrt[N]{2}} \sqrt[N]{r^{N}+1}, \quad 0 \leq r \leq 1,
$$


which are the convex functions considered in [1] with $\varepsilon=1 / 2$. Then, $\bar{v}_{\lambda} \in \mathcal{A}(\lambda)$ for every $\lambda>1$ and, moreover

$\bar{v}_{\lambda}^{\prime}\left(\frac{\bar{v}_{\lambda}}{r}\right)^{N-1}=\frac{\lambda^{N}}{2}, \quad \bar{v}_{\lambda}^{\prime}(r) \leq \bar{v}_{\lambda}^{\prime}(1)=\frac{\lambda}{2} \quad$ and $\quad \bar{v}_{\lambda}(r) \leq \bar{v}_{\lambda}(1)=\lambda$.

We claim that $J_{S}\left(\bar{v}_{\lambda}\right)-J_{S}\left(v_{\lambda}\right) \rightarrow-\infty$ as $\lambda \rightarrow+\infty$. We have

$$
J_{S}\left(\bar{v}_{\lambda}\right)-J_{S}\left(v_{\lambda}\right)=I_{1}+I_{2}+w^{\infty} \sigma_{N}\left(\frac{\lambda}{\sqrt[N]{2}}\right)^{N-1}
$$

where $I_{1}$ and $I_{2}$ are the integrals involving $\Phi_{1}$ and $\Phi_{2}$ respectively. As to the first summand, from (H2), (H3) and (4.5) we deduce

$$
\left|I_{1}\right| \leq \sigma_{N} \int_{0}^{1}\left[\Phi^{1}\left(\bar{v}_{\lambda}^{\prime}, \frac{\bar{v}_{\lambda}}{r}\right)+\Phi^{1}(\lambda, \lambda)\right] r^{N-1} d r \leq C\left(1+\lambda^{p}\right)
$$

for some constant $C$ independent of $\lambda$. Setting $w(t)=w^{\infty} t[1+\bar{w}(t)], t>0$, as in the proof of the previous theorem, for the second summand we obtain from (4.5)

$$
\begin{aligned}
I_{2} & =\sigma_{N} \int_{0}^{1}\left[w\left(\frac{\lambda^{N}}{2}\right)-w\left(\lambda^{N}\right)\right] r^{N-1} d r=\frac{\sigma_{N}}{N}\left[w\left(\frac{\lambda^{N}}{2}\right)-w\left(\lambda^{N}\right)\right] \\
& =-\frac{\sigma_{N}}{N} w^{\infty} \frac{\lambda^{N}}{2}\left[1-\bar{w}\left(\frac{\lambda^{N}}{2}\right)+2 \bar{w}\left(\lambda^{N}\right)\right] .
\end{aligned}
$$

Since $\bar{w}(t) \rightarrow 0$ as $t \rightarrow \infty$ and $1<p<N$, the claim is proved.

In addition, exploiting again the arguments used above for $J_{V}$ and $J$, we find that the EL equation for $J_{S}$ is given by (3.11) with the boundary conditions

$$
\left\{\begin{array} { l } 
{ v ( 0 ) = 0 } \\
{ v ( 1 ) = \lambda }
\end{array} \text { or } \quad \left\{\begin{array}{l}
v(0)>0 \text { and } \lim _{r \rightarrow 0^{+}} T(r)=\frac{(N-1) w^{\infty}}{v(0)} \\
v(1)=\lambda
\end{array}\right.\right.
$$

Moreover, every solution $v$ to $(3.11)$ is smooth on $(0,1]$ and the unique solution which is nonsingular at $r=0$ is the linear solution $v_{\lambda}$ for every $\lambda \geq 1$. Thus, cavitation occurs for every large enough $\lambda$ and we have thus proved the following result.

Theorem 4.3. Assume that $(\mathrm{H} 1), \ldots,(\mathrm{H} 8)$ hold. Every minimizer $v \in \mathcal{A}(\lambda)$ of $J_{S}$ satisfies $v(0)>0$ for every large enough $\lambda>1$.

\section{The model case for $J_{S}$}

In this part we investigate the properties of the solutions to the EL equation (3.11) with the boundary condition (4.6) for $J_{S}$ in the $3 \mathrm{D}$ model case corresponding to $\theta(t)=t^{2}$ and $w(t)=t+1 / t, t>0$, i.e. to the energy density

$$
W(A)=|A|^{2}+\operatorname{det} A+\frac{1}{\operatorname{det} A}, \quad A \in \mathbb{M}^{3 \times 3} \text { with } \operatorname{det} A>0 .
$$

We shall exploit the shooting method again. In this model case, the hypotheses $(\mathrm{H} 1), \ldots,(\mathrm{H} 8)$ are satisfied with $p=2$ and $w^{\infty}=1$. Thus, Corollary 3.4 
holds and radial minimizers of $J_{S}$ exist and are of class $\mathcal{C}^{2}$ and satisfy the EL equation

$$
r\left[1+\frac{r^{2}}{\left(v^{\prime}\right)^{3} v^{2}}\right] v^{\prime \prime}=2\left(\frac{v}{r}-v^{\prime}\right)\left[1+\frac{r^{3}}{\left(v^{\prime}\right)^{2} v^{3}}\right]
$$

for every $r \in(0,1]$, with boundary conditions

$$
\left\{\begin{array} { l } 
{ v ( 0 ) = 0 } \\
{ v ( 1 ) = \lambda }
\end{array} \text { or } \quad \left\{\begin{array}{l}
v(0)>0 \text { and } \lim _{r \rightarrow 0^{+}} T(r)=\frac{2}{v(0)} \\
v(1)=\lambda
\end{array}\right.\right.
$$

where $T$ is given by

$$
T(r)=1+\left(\frac{r}{v}\right)^{2}\left[2 v^{\prime}-\left(\frac{r}{v}\right)^{2} \frac{1}{\left(v^{\prime}\right)^{2}}\right]
$$

Note that, as the derivative of every singular solution $v$ to (5.1) is bounded by $v^{\prime}(1)<\lambda$ because of (c) of Lemma 3.6, it follows that

$$
\lim _{r \rightarrow 0^{+}} T(r)=\frac{2}{v(0)} \quad \text { if and only if } \lim _{r \rightarrow 0^{+}}\left(\frac{r}{v}\right)^{4} \frac{1}{\left(v^{\prime}\right)^{2}}=1-\frac{2}{v(0)},
$$

which yields $v(0) \geq 2$. Thus, the radius of possible cavities of singular solutions to the EL equation (5.1), (5.2) must be at least 2 .

By means of the change of variables defined by

$$
t=\frac{v(r)}{r} \quad \text { and } \quad q(t)=v^{\prime}(r)
$$

(see Lemma 4 in [10]), the second order differential equation (5.1) becomes the first order differential equation

$$
q^{\prime}=-2 \frac{q}{t} \frac{1+q^{2} t^{3}}{1+q^{3} t^{2}}
$$

and the corresponding Cauchy problem with initial condition

$$
q(\lambda)=\alpha
$$

has a solution $q_{\lambda, \alpha}(t)$ defined for $t \geq \lambda$ for every choice of $\alpha>0$. Moreover, if $v_{\lambda, \alpha}$ is a solution to the Cauchy problem (5.1) with initial values $v(1)=\lambda$ and $v^{\prime}(1)=\alpha$ with $0<\alpha<\lambda$, then the function obtained from $v_{\lambda, \alpha}(r)$ by the change of variables (5.4) is the solution $q_{\lambda, \alpha}$ to the differential equation (5.5) with initial value (5.6) and viceversa.

Lemma 5.1. The solutions $q_{\lambda, \alpha}$ to (5.5), (5.6) have the following properties:

(a) $q_{\lambda, \alpha_{1}}(t)<q_{\lambda, \alpha_{2}}(t)<t$ for every $0<\alpha_{1}<\alpha_{2} \leq \lambda$ and $t \geq \lambda$;

(b) $\lim _{t \rightarrow+\infty} q_{\lambda, \alpha}(t)=0$ for every $0<\alpha \leq \lambda$;

(c) the function $t \mapsto\left[q_{\lambda, \alpha_{2}}(t)-q_{\lambda, \alpha_{1}}(t)\right]$ is decreasing for $t \geq \lambda$ and for every $0<\alpha_{1}<\alpha_{2} \leq \lambda$.

Proof. It is

$$
q_{\lambda, \alpha_{1}}(\lambda)=\alpha_{1}<q_{\lambda, \alpha_{2}}(\lambda)=\alpha_{2} \leq \lambda
$$


for $0<\alpha_{1}<\alpha_{2} \leq \lambda$. Thus, (a) follows from uniqueness of solutions to (5.5) and (5.6). Moreover, as the right hand side of (5.5) is negative, it is easy to conclude that (b) holds.

Now, we are left to prove (c). To simplify the notation, set $q_{i}=q_{\lambda, \alpha_{i}}$ for $i=1,2$. Since $q_{1}$ and $q_{2}$ are solution to (5.5), we have

$$
\left[q_{2}(t)-q_{1}(t)\right]^{\prime}=H\left(q_{2}(t), t\right)-H\left(q_{1}(t), t\right) \quad \text { where } H(q, t)=-2 \frac{q+q^{3} t^{3}}{t+q^{3} t^{3}}
$$

and

$$
H_{q}(q, t)=-\frac{2 t}{\left(t+q^{3} t^{3}\right)^{2}}\left[1+q^{2} t^{2}(3 t-2 q)\right]<0, \quad 0<q<t .
$$

Then, (a) implies that $\left[q_{2}(t)-q_{1}(t)\right]^{\prime}<0$ for all $t \geq \lambda$ whence (c) follows.

Now, let $T(\lambda, \alpha)(r)$ be the value of $T(r)$ in (5.3) when $v=v_{\lambda, \alpha}$ and set

$$
\tau(\lambda, \alpha)=\lim _{r \rightarrow 0^{+}} T(\lambda, \alpha)(r) \quad(\lambda, \alpha) \in D,
$$

where $D=\{(\lambda, \alpha): 0<\alpha<\lambda\}$. We want to show that, for large enough $\lambda$, there exists a value $\alpha<\lambda$, such that $\tau(\lambda, \alpha)=2 /[v(\lambda, \alpha)(0)]$ and that, contrary to the superlinear case considered by Stuart, there must be at least two values of $\alpha$ with this property. To do this, we prove the following properties of $\tau(\lambda, \alpha)$.

Lemma 5.2. Let $\tau: D \rightarrow \mathbb{R}$ be the function defined by (5.7). Then,

(a) $\tau$ is continuous on $D$;

(b) $\tau(\lambda, \cdot):(0, \lambda) \rightarrow \mathbb{R}$ is strictly increasing for every $\lambda>0$;

(c) $\tau(\cdot, \alpha):(\alpha,+\infty) \rightarrow \mathbb{R}$ is strictly increasing for every $\alpha>0$;

(d) $\lim _{\alpha \rightarrow 0^{+}} \tau(\lambda, \alpha)=-\infty$ for every $\lambda>0$.

Proof. Since $v=v(\lambda, \alpha)$ is a solution to (5.1), for every $s \in(0,1]$ we have

$$
\begin{aligned}
T(\lambda, \alpha)(s) & =T(\lambda, \alpha)(1)+\int_{1}^{s} \frac{\mathrm{d}}{\mathrm{d} r} T(\lambda, \alpha)(r) d r \\
& =1+\frac{1}{\lambda^{2}}\left(2 \alpha-\frac{1}{\lambda^{2} \alpha^{2}}\right)-4 \int_{1}^{s}\left(\frac{r}{v}\right)^{3}\left(v^{\prime}+\frac{v}{r}\right)\left(\frac{v}{r}\right)^{\prime} d r .
\end{aligned}
$$

By the change of variables (5.4), we have

$$
T(\lambda, \alpha)(s)=1+\frac{2 \lambda^{2} \alpha^{3}-1}{\lambda^{4} \alpha^{2}}-4 \int_{\lambda}^{v(s) / s} \frac{q(t)+t}{t^{3}} d t
$$

where $q(t)=q_{\lambda, \alpha}(t), t \geq \lambda$.

In view of (a) of Lemma 5.1 , we have $[q(t)+t] / t^{3}<2 / t^{2}$ for every $t \geq \lambda$. Thus, we can pass to the limit within the integral and we obtain

$$
\tau(\lambda, \alpha)=1+\frac{2 \lambda^{2} \alpha^{3}-1}{\lambda^{4} \alpha^{2}}-4 \int_{\lambda}^{+\infty} \frac{q(t)+t}{t^{3}} d t .
$$

Therefore, (a) follows from the continuous dependence of solutions to (5.5), (5.6) on the data $\alpha$ and $\lambda$ and from the dominated convergence theorem.

To prove (b), choose $0<\alpha_{1}<\alpha_{2}<\lambda$ and set $q_{i}=q_{\lambda, \alpha_{i}}, i=1,2$. By definition of $\tau$, we have 


$$
\tau\left(\lambda, \alpha_{2}\right)-\tau\left(\lambda, \alpha_{1}\right)=\frac{2 \lambda^{2} \alpha_{2}{ }^{3}-1}{\lambda^{4} \alpha_{2}{ }^{2}}-\frac{2 \lambda^{2} \alpha_{1}{ }^{3}-1}{\lambda^{4} \alpha_{1}{ }^{2}}-4 \int_{\lambda}^{+\infty} \frac{q_{2}(t)-q_{1}(t)}{t^{3}} d t
$$

Hence, by (c) of Lemma 5.1, we have that $q_{2}(t)-q_{1}(t) \leq q_{2}(\lambda)-q_{1}(\lambda)=\alpha_{2}-\alpha_{1}$ for $t \geq \lambda$. Thus,

$$
\begin{aligned}
\tau\left(\lambda, \alpha_{2}\right)-\tau\left(\lambda, \alpha_{1}\right) & \geq \frac{2}{\lambda^{2}}\left(\alpha_{2}-\alpha_{1}\right)+\frac{1}{\lambda^{4}}\left(\frac{1}{\alpha_{1}^{2}}-\frac{1}{\alpha_{2}^{2}}\right)-4 \int_{\lambda}^{+\infty} \frac{\alpha_{2}-\alpha_{1}}{t^{3}} d t \\
& =\frac{1}{\lambda^{4}}\left(\frac{1}{\alpha_{1}^{2}}-\frac{1}{\alpha_{2}^{2}}\right)>0
\end{aligned}
$$

and (b) is proved.

As to (c), by the change of variables $(5.4)$, for $(\lambda, \alpha) \in D$ we can write

$\tau(\lambda, \alpha)=\lim _{r \rightarrow 0^{+}}\left\{1-\left(\frac{r}{v_{\lambda, \alpha}(r)}\right)^{4} \frac{1}{\left(v_{\lambda, \alpha}^{\prime}(r)\right)^{2}}\right\}=\lim _{t \rightarrow+\infty}\left\{1-\frac{1}{t^{4}\left[q_{\lambda, \alpha}(t)\right]^{2}}\right\}$.

Now, given $\lambda_{1}>\lambda$, the equality

$$
q_{\lambda, \alpha}(t)=q_{\lambda_{1}, \alpha_{1}}(t), \quad t \geq \lambda_{1},
$$

holds for $\alpha_{1}=q_{\lambda, \alpha}\left(\lambda_{1}\right)$. Therefore,

$$
\begin{aligned}
\tau(\lambda, \alpha) & =\lim _{t \rightarrow+\infty}\left\{1-\frac{1}{t^{4}\left[q_{\lambda, \alpha}(t)\right]^{2}}\right\}=\lim _{t \rightarrow+\infty}\left\{1-\frac{1}{t^{4}\left[q_{\lambda_{1}, \alpha_{1}}(t)\right]^{2}}\right\} \\
& =\tau\left(\lambda_{1}, \alpha_{1}\right)<\tau\left(\lambda_{1}, \alpha\right)
\end{aligned}
$$

where the last inequality is due to (b) and to the fact that it is $\alpha_{1}=q_{\lambda, \alpha}\left(\lambda_{1}\right)<$ $q_{\lambda, \alpha}(\lambda)=\alpha$. This concludes the proof of (c).

Finally, (d) follows immediately from (5.8).

Lemma 5.3. Let $g:(0,+\infty) \rightarrow \mathbb{R}$ be the function defined by

$$
g(\lambda)=\lim _{\alpha \rightarrow \lambda^{-}} \tau(\lambda, \alpha), \quad \lambda>0 .
$$

Then,

(a) $g$ is continuous;

(b) $\lim _{\lambda \rightarrow 0^{+}} g(\lambda)=-\infty$ and $\lim _{\lambda \rightarrow+\infty} g(\lambda)=1$;

(c) $g$ is strictly increasing.

Proof. From (5.8), the continuous dependence of solutions to (5.5), (5.6) on the data $\alpha$ and $\lambda$ and from the dominated convergence theorem, we obtain

$$
g(\lambda)=1+\frac{2 \lambda^{5}-1}{\lambda^{6}}-4 \int_{\lambda}^{+\infty} \frac{q_{\lambda, \lambda}(t)+t}{t^{3}} d t, \quad \lambda>0
$$

where $q_{\lambda, \lambda}$ is the solution to (5.5) corresponding to the initial value $q(\lambda)=\lambda$. Therefore, the continuity of $g$ is a consequence of the continuous dependence of $q_{\lambda, \lambda}$ on $\lambda$ and also the limits in (b) follow immediately from the integral representation of $g$.

As to (c), consider $1 \leq \lambda_{1}<\lambda_{2}$. By Lemma 5.2 for every $\alpha_{1}<\lambda_{1}$ and for every $\alpha_{2} \in\left(\lambda_{1}, \lambda_{2}\right)$, we have 


$$
\tau\left(\lambda_{1}, \alpha_{1}\right)<\tau\left(\lambda_{2}, \alpha_{1}\right)<\tau\left(\lambda_{2}, \alpha_{2}\right)<g\left(\lambda_{2}\right) .
$$

Then, $g\left(\lambda_{1}\right)=\sup \left\{\tau\left(\lambda_{1}, \alpha_{1}\right): \alpha_{1} \in\left(0, \lambda_{1}\right)\right\}<g\left(\lambda_{2}\right)$ and also (c) is proved.

Then, we consider the continuous function $\alpha \in(0, \lambda) \mapsto v_{\lambda, \alpha}(0)$. It is clear that $v_{\lambda, \alpha}(0) \rightarrow 0^{+}$as $\alpha \rightarrow \lambda^{-}$and $v_{\lambda, \alpha}(0) \rightarrow \lambda^{-}$as $\alpha \rightarrow 0^{+}$because $v_{\lambda, \alpha}$ is convex and hence $\lambda>v_{\lambda, \alpha}(0)>\lambda-\alpha$ for $0<\alpha<\lambda$. Thus,

$$
0<\frac{2}{\lambda}<\frac{2}{v_{\lambda, \alpha}(0)}<\frac{2}{\lambda-\alpha}, \quad 0<\alpha<\lambda .
$$

Since $g(\lambda)<1$ this shows that for $\lambda \geq 1$ sufficiently close to 1 there is no $0<\alpha<\lambda$ such that $\tau(\alpha, \lambda)=2 / v_{\lambda, \alpha}(0)$, i.e. the linear function $v_{\lambda}$ is the only solution to the EL equation (5.1), (5.2).

Finally, we prove that for every $\lambda$ large enough, there are at least two solutions to (5.1), (5.2) which are singular at $r=0$. In fact, recalling that $g(\lambda) \rightarrow 1^{-}$ as $\lambda \rightarrow+\infty$, we can choose $\lambda_{0}>1$ and $0<\alpha_{0}<\lambda_{0}$ in such a way that $\tau\left(\alpha_{0}, \lambda_{0}\right)>1 / 2$. Then, the monotonicity of $\tau$ with respect to $\lambda$ implies that $\tau\left(\alpha_{0}, \lambda\right) \geq \tau\left(\alpha_{0}, \lambda_{0}\right)>1 / 2$ for $\lambda>\lambda_{0}$ and we can assume also that $2 / v_{\lambda, \alpha_{0}}(0)<2 /\left(\lambda-\alpha_{0}\right)<1 / 2$ for $\lambda>\lambda_{0}$. Moreover, $\tau(\alpha, \lambda) \rightarrow-\infty$ as $\alpha \rightarrow 0^{+}$by (d) of Lemma 5.2 whereas $2 / v_{\lambda, \alpha}(0)$ tends to $2 / \lambda$ as $\alpha \rightarrow 0^{+}$and to $+\infty$ as $\alpha \rightarrow \lambda^{-}$for every fixed $\lambda>0$. Thus, for every $\lambda>\lambda_{0}$, there are at least two distinct values $0<\alpha_{1}<\alpha_{2}<\lambda$ such that

$$
\tau\left(\alpha_{i}, \lambda\right)=\frac{2}{v_{\lambda, \alpha_{i}}(0)}, \quad i=1,2,
$$

and the corresponding $v_{\lambda, \alpha_{i}}$ are singular solutions to the EL equation (5.1), (5.2).

Summing up, we have thus proved that there are values $1<\lambda_{c}^{-} \leq \lambda_{c}^{+}$such that the linear function $v_{\lambda}$ is the unique minimizer of $J_{S}$ for $1 \leq \lambda<\lambda_{c}^{-}$, whereas $J_{S}$ has a minimizer $v$ with $v(0)>0$ for every $\lambda>\lambda_{c}^{+}$. As explained before, for $\lambda$ in the possible, intermediate range between $\lambda_{c}^{-}$and $\lambda_{c}^{+}$, our analysis only proves that, besides the linear function, there are other solutions to the EL equation (5.1), (5.2) which are singular at $r=0$ but does not yield any information whether the minimizer is the linear function $v_{\lambda}$ or any of the singular solutions, though the obvious conjecture is that $\lambda_{c}^{-}=\lambda_{c}^{+}$.

\section{Acknowledgements}

We wish to thank P. Marcellini for introducing us to this subject and D. Mucci for many helpful discussions.

\section{References}

[1] Ball, J.M.: Discontinuous equilibrium solutions and cavitation in nonlinear elasticity. Philos. Trans. R. Soc. Lond. Ser. A 306(1496), 557-611 (1982)

[2] Blatz, P.J., Ko, W.L.: Application of finite elastic theory to the deformation of rubbery materials. Trans. Soc. Rheol. 6, 223-251 (1962) 
[3] Giaquinta, M., Modica, G., Souček, J.: Cartesian currents in the calculus of variations. I. In: Ergebnisse der Mathematik und ihrer Grenzgebiete. 3. Folge, Vol. 37. Springer-Verlag, Berlin (1998)

[4] Giaquinta, M., Modica, G., Souček, J.: Cartesian currents in the calculus of variations. II. In: Ergebnisse der Mathematik und ihrer Grenzgebiete. 3. Folge, Vol. 38. Springer-Verlag, Berlin (1998)

[5] Horgan, C.O., Polignone, D.A.: Cavitation in nonlinearly elastic solids: a review. Appl. Mech. Rev. 48(8), 471-485 (1995)

[6] Marcellini, P.: The stored-energy for some discontinuous deformations in nonlinear elasticity. In: Partial differential equations and the calculus of variations, Vol. II, Progr. Nonlinear Differential Equations Appl., Vol. 2, Birkhäuser Boston, Boston, MA, pp. 767-786 (1989)

[7] Müller, S., Spector, S.J.: An existence theory for nonlinear elasticity that allows for cavitation. Arch. Ration. Mech. Anal. 131(1), 1-66 (1995)

[8] Podio-Guidugli, P., Vergara Caffarelli, G., Virga, E.G.: Discontinuous energy minimizers in nonlinear elastostatics: an example of J. Ball revisited. J. Elast. 16, 75-96 (1986)

[9] Sivaloganathan, J.: Uniqueness of regular and singular equilibria for spherically symmetric problems of nonlinear elasticity. Arch. Ration. Mech. Anal. 96(2), 97$136(1986)$

[10] Stuart, C.A.: Radially symmetric cavitation for hyperelastic materials. Ann. Inst. H. Poincaré Anal. Non Linéaire 2(1), 33-66 (1985)

Pietro Celada

Dipartimento di Matematica

Università degli Studi di Parma

V.le G. B. Usberti 53/A

43124 Parma

Italy

e-mail: pietro.celada@unipr.it

Stefania Perrotta

Dipartimento di Matematica Pura ed Applicata "G. Vitali"

Università degli Studi di Modena e Reggio Emilia

Via Campi 213/B

41125 Modena

Italy

e-mail: perrotta@unimore.it

Received: 2 December 2011.

Accepted: 10 July 2012. 